\title{
Recoded organisms engineered to depend on synthetic amino acids
}

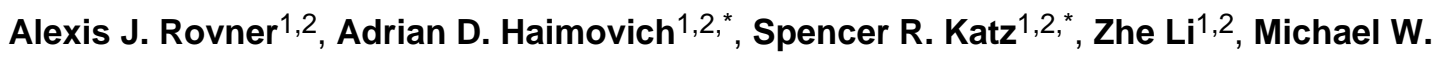 \\ Grome $^{1,2}$, Brandon M. Gassaway ${ }^{2,3}$, Miriam Amiram ${ }^{1,2}$, Jaymin R. Patel ${ }^{1,2}$, Ryan R. \\ Gallagher $^{1,2}$, Jesse Rinehart ${ }^{2,3}$, and Farren J. Isaacs ${ }^{1,2}$ \\ ${ }^{1}$ Department of Molecular, Cellular and Developmental Biology, Yale University, New Haven, CT \\ 06520, USA \\ ${ }^{2}$ Systems Biology Institute, Yale University, West Haven, CT 06516, USA \\ ${ }^{3}$ Department of Cellular and Molecular Physiology, Yale University, New Haven, CT 06520, USA
}

\section{Abstract}

Genetically modified organisms (GMOs) are increasingly used in research and industrial systems to produce high-value pharmaceuticals, fuels, and chemicals ${ }^{1}$. Genetic isolation and intrinsic biocontainment would provide essential biosafety measures to secure these closed systems and enable safe applications of GMOs in open systems ${ }^{2,3}$, which include bioremediation ${ }^{4}$ and probiotics ${ }^{5}$. Although safeguards have been designed to control cell growth by essential gene regulation ${ }^{6}$, inducible toxin switches ${ }^{7}$, and engineered auxotrophies ${ }^{8}$, these approaches are compromised by cross-feeding of essential metabolites, leaked expression of essential genes, or genetic mutations ${ }^{9,10}$. Here, we describe the construction of a series of genomically recoded organisms (GROs) ${ }^{11}$ whose growth is restricted by the expression of multiple essential genes that depend on exogenously supplied synthetic amino acids (sAAs). We introduced a Methanocaldococcus jannaschii tRNA:aminoacyl-tRNA synthetase (aaRS) pair into the chromosome of a GRO that lacks all TAG codons and release factor 1, endowing this organism with the orthogonal translational components to convert TAG into a dedicated sense codon for sAAs. Using multiplex automated genome engineering (MAGE) ${ }^{12}$, we introduced in-frame TAG codons into 22 essential genes, linking their expression to the incorporation of synthetic phenylalanine-derived amino acids. Of the $60 \mathrm{sAA}$-dependent variants isolated, a notable strain harboring 3 TAG codons in conserved functional residues ${ }^{13}$ of MurG, DnaA and SerS and containing targeted tRNA deletions maintained robust growth and exhibited undetectable escape frequencies upon culturing $\sim 10^{11}$ cells on solid media for seven days or in liquid media for 20 days. This is a significant improvement over existing biocontainment approaches ${ }^{2,3,6-10}$. We

Reprints and permissions information is available at www.nature.com/reprints

Correspondence and requests for materials should be addressed to F.J.I. (farren.isaacs@yale.edu).

These authors contributed equally to this work

Supplementary Information: is linked to the online version of the paper at www.nature.com/nature.

Author Contributions A.J.R and F.J.I conceived the study, designed experiments and wrote the paper with assistance from A.D.H. and S.R.K.; A.J.R. conducted experiments with assistance from S.R.K., A.D.H., M.G., M.A., J.R.P. and R.R.G.; B.G. and J.R. conducted mass spectrometry; All authors commented on the paper and F.J.I. supervised all aspects of the study.

A.J.R. and F.J.I. have filed a provisional application with the US Patent and Trademark Office on this work. 
constructed synthetic auxotrophs dependent on sAAs that were not rescued by cross-feeding in environmental growth assays. These auxotrophic GROs possess alternate genetic codes that impart genetic isolation by impeding horizontal gene transfer ${ }^{11}$ and now depend on the use of synthetic biochemical building blocks, advancing orthogonal barriers between engineered organisms and the environment.

The advent of recombinant DNA technologies in the 1970s established genetic cloning methods ${ }^{14}$, ushering in the era of biotechnology. Over the past decade, synthetic biology has fueled the emergence of GMOs with increased sophistication as common and valued solutions in clinical, industrial, and environmental settings ${ }^{1,4,5}$, necessitating the development of safety and security measures first outlined in the 1975 Asilomar conference on recombinant $\mathrm{DNA}^{15}$. While guidelines for physical containment and safe use of organisms have been widely adopted, intrinsic biocontainment - biological barriers limiting the spread and survival of microorganisms in natural environments - remains a defining challenge. Existing biocontainment strategies employ natural auxotrophies or conditional suicide switches where top safeguards meet the $10^{-8} \mathrm{NIH}$ standard ${ }^{16}$ for escape frequencies (EFs), but can be compromised by metabolic cross-feeding or genetic mutation ${ }^{9,10}$. We hypothesized that engineering dependencies on synthetic biochemical building blocks would enhance existing containment strategies by establishing orthogonal barriers not feasible in organisms with a standard genetic code.

Our approach to engineering biocontainment utilized a GRO lacking all instances of the TAG codon and release factor 1 (terminates translation at UAA and UAG), eliminating termination of translation at UAG and endowing the organism with increased viral resistance, a common form of horizontal gene transfer (HGT). The TAG codon was then converted to a sense codon through the introduction of an orthogonal translation system (OTS) containing an aaRS:tRNA pair, permitting site-specific incorporation of sAAs into proteins without impairing cellular fitness ${ }^{11}$. Leveraging these unique properties of the GRO, we sought to reintroduce the TAG codon into essential genes to restrict growth to defined media containing sAAs. We also eliminated the use of multi-copy plasmids, which reduce viability and growth ${ }^{17}$, impose biosynthetic burden, persist poorly in host cells over time $^{18}$, and increase the risk of acquiring genetic escape mutants $(\mathrm{EMs})^{3}$, by manipulating native chromosomal essential genes and integrating the OTS into the genome. To engineer synthetic auxotrophies, we chose essential genes of varying expression levels (Methods), many of whose functions (e.g., replication, translation) cannot be complemented by crossfeeding of metabolites. Genes dispersed throughout the genome were selected to prevent a single HGT event from compromising containment.

We pursued three strategies to engineer dependence on nontoxic, membrane permeable, and well-characterized sAAs through the introduction of TAG codons into essential genes: (1) insertion at the amino-terminus, (2) substitution of residues with computationally predicted tolerances ${ }^{19}$, and (3) substitution of conserved ${ }^{13}$ residues at functional sites (Fig. 1a). We initially pursued the first two strategies in a GRO containing an OTS optimized for the sAA p-acetyl-ı-phenylalanine (pAcF, a). Using MAGE ${ }^{12}$, we targeted 155 codons for TAG incorporation via four pools of oligonucleotides (Supplementary Table 1, Supplementary 
Table 2) in permissive media containing pAcF and $\mathrm{L}$-arabinose (aaRS induction) (Fig. 1b). After replica plating on nonpermissive media lacking $\mathrm{pAcF}$ and $\mathrm{L}$-arabinose, we isolated eight pAcF auxotrophs with one strain containing two TAGs in essential genes (Fig. 1c and Supplementary Table 3). To determine whether our strategy was capable of creating synthetic auxotrophs dependent on other sAAs, MAGE was used to mutagenize annotated residues in the sAA binding pocket of the pAcF aaRS (Supplementary Table 4) to accommodate p-iodo-s-phenylalanine (pIF, $\beta$ ) or p-azido-s-phenyalanine (pAzF, $\gamma$ ) in two strains. After MAGE-based incorporation of TAGs and selections on permissive and nonpermissive solid media, we obtained eight pIF and $23 \mathrm{pAzF}$ auxotrophs harboring one to four TAGs at 30 distinct loci across 20 essential genes (Supplementary Table 3, Supplementary Table 5). Together, these data demonstrate the modularity of our approach and that synthetic auxotrophs can be engineered across many essential genes using multiple sAAs (Extended Data Fig. 1).

Doubling time (DT) measurements in permissive media revealed minimal or no fitness impairment of synthetic auxotrophs relative to their non-contained ancestors with a genomically integrated OTS (Fig. 2a and Supplementary Table 6). To quantify the degree of containment, we measured the ratio of colony forming units (CFUs) on nonpermissive to permissive solid media and observed a range of EFs spanning $10^{-3}$ to $10^{-7}$ (Fig. 2b). One notable strain DnaX.Y113a, preserved the DT of its non-contained ancestor (Fig. 2a) while maintaining an $\mathrm{EF}$ of $6.7 \times 10^{-7}$ (Fig. 2b). We directly investigated pAcF incorporation in DnaX.Y113a using mass spectrometry and identified peptides containing pAcF at Y113 (Fig. 2c).

To investigate escape mechanisms of EMs derived from synthetic auxotrophs with one essential TAG codon, we performed targeted sequencing and observed transition mutations $(\mathrm{A} \bullet \mathrm{T} \rightarrow \mathrm{G} \bullet \mathrm{C}$ and $\mathrm{G} \bullet \mathrm{C} \rightarrow \mathrm{A} \bullet \mathrm{T})$ commonly observed in mismatch repair-deficient strains $(\Delta m u t S)^{20}$. All isolated DnaX.Y113aEMs incorporate tryptophan by mutation of the TAG codon to TGG. SecY.Y122a EMs incorporate glutamine by mutation of $g \ln V$ to form a glutamine amber suppressor or mutation of the secY.Y122 TAG codon to CAG (Supplementary Table 7). One of three SecY.Y122a EMs was wild type at the secY.Y122 TAG codon and putative amber suppressor loci ${ }^{21}$, but whole genome sequencing (Supplementary Table 8) revealed a Q54D missense mutation in rpsD (30S ribosomal subunit S4). This site is implicated in ribosome fidelity 22,23 and is the causal mutation leading to escape in this mutant (Extended Data Fig. 2).

These escape mechanisms informed two sets of experiments to engineer strains with lower EFs. First, we sought to create synthetic auxotrophs with increased numbers of TAGs (Fig. 2d) by combining TAGs from strains possessing the lowest EFs (i.e., dnaX.Y113, lspA.Y54, and $\sec Y$.Y122) into a single strain. In strains containing two TAGs, the EF was reduced to $1.4 \times 10^{-7}$ (rEc. $\gamma . \mathrm{dB} .26$ ) and $1.4 \times 10^{-8}$ (rEc.ß.dB.9) (strain annotations are listed in Supplementary Table 6). In strains containing three TAGs, EFs were further reduced to

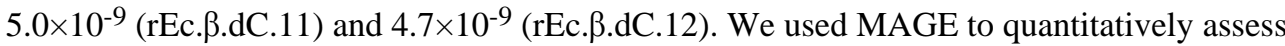
the effects of non-synonymous mutations at individual TAG codons in strains incorporating pIF at SecY.Y122, DnaX.Y113, and LspA.Y54 by mutating the TAG site to sense codons for all 20 natural amino acids. Strains containing multiple TAGs were less likely to survive 
when one TAG was compromised (Extended Data Fig. 3). In a second set of experiments, we restored $m u t S$ and observed a decreased EF in strains by 1.5- to 3.5-fold (Fig. 2e, Supplementary Table 6). EMs derived from $m u t S^{+}$higher-order TAG strains exhibited impaired fitness with 1.14- to 1.28-fold greater DTs than their contained ancestors. Whole genome sequencing was performed on these EMs and revealed mutations of tyrosine tRNAs to form tyrosine amber (UAG) or ochre (UAA) suppressors (Supplementary Table 9).

To reduce EFs below $\sim 10^{-9}$ and eliminate rescue by natural amino acids, we pursued a third strategy to replace conserved and functional residues in essential proteins with sAAs (Fig. 1a). Using the Conserved Domain Database ${ }^{13}$, we searched all essential proteins for tyrosine, tryptophan, and phenylalanine residues involved in protein-protein interactions (e.g., dimerization) or located within active sites to identify candidates suitable for replacement with phenylalanine-derived sAAs. After targeted insertion of TAG codons using MAGE, we isolated four synthetic auxotrophs with pAzF incorporated at GlyQ.Y226 (glycyl-tRNA synthetase a subunit, dimer interface), Lnt.Y388 (apolipoprotein Nacyltransferase, active site), MurG.F243 (N-acetylglucosaminyl transferase, active site), and DnaA.W6 (chromosomal replication initiator protein, oligomerization site ${ }^{24}$ ) in strains with minor fitness impairments (Fig. 3a) and EFs spanning $10^{-5}$ to $10^{-7}$ (Fig. 3b). Identical experiments to incorporate $\mathrm{pAcF}$ and $\mathrm{pIF}$ failed to generate synthetic auxotrophs, suggesting that the targeted residues are recalcitrant to replacement by pAcF and pIF. Since pAzF was able to replace conserved and functional tyrosine, phenylalanine and tryptophan residues across several essential proteins, we hypothesized that engineering strains to contain higherorder TAG combinations would limit escape by mutations that cause incorporation of natural amino acids at multiple TAG codons. EFs of $1.6 \times 10^{-9}$ and $2.3 \times 10^{-9}$ were observed for strains containing two TAG codons:rEc. $\gamma . d B .41$ (DnaA.W6 and MurG.F243) and rEc. $\gamma . \mathrm{dB} .43$ (DnaA.W6 and SerS.F213), respectively. Upon restoring $m u t S$, the EF of rEc. $\gamma . \mathrm{dB} .41^{\prime}$ fell to $6.0 \times 10^{-10}$ (Fig. 3b). Merging all three sites into one strain (rEc. $\gamma . \mathrm{dC} .46$ ) and its $m u t S^{+}$derivative (rEc. $\gamma . \mathrm{dC} .46^{\prime}$ ) led to EFs of $<7.9 \times 10^{-11}$ and $<4.4 \times 10^{-11}$ (below the detection limit of our plate-based assay), respectively (Supplementary Table 6).

Temporal monitoring of rEc. $\gamma \cdot \mathrm{dC} .46^{\prime}$ revealed the emergence of growth-impaired EMs two days post-plating on nonpermissive solid media (Fig. 3d). Sequencing of EMs derived from strains rEc. $\gamma . \mathrm{dC} .41^{\prime}$ and $\mathrm{rEc} . \gamma \cdot \mathrm{dC} .46^{\prime}$ revealed amber suppressor-forming mutations at one of three tyrosine tRNAs (tyrT, tyr $V$, tyr $U$ ) with growth impairments spanning 1.61- to 2.10fold increase in DT relative to their contained ancestors (Supplementary Table 9, Supplementary Table 10). Given that $E$. coli contains three tyrosine tRNAs, we hypothesized that deletion of tyrT and $t y r V^{25}$ would prevent acquisition of amber suppressor-forming mutations at tyr $U$, as preservation of this single remaining copy of tRNA $^{\mathrm{Tyr}}$ would be required to maintain fidelity of tyrosine incorporation during protein

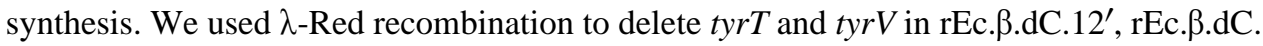
$12^{\prime}$.E7 (EM of rEc. $\beta . \mathrm{dC} .12^{\prime}$ ), and rEc. $\gamma . \mathrm{dC} .46^{\prime}$ with a chloramphenicol resistance gene. Deletion of tyrT and $t y r V$ restored containment of the EM, establishing the causal escape mechanism (Extended Data Fig. 4). Moreover, tyrT/V deletions in rEc. $\beta . d C .12^{\prime} . \Delta \mathrm{tY}$ and rEc. $\gamma . \mathrm{dC} .46^{\prime} . \Delta \mathrm{tY}$ decreased EFs below detectable levels $\left(<4.9 \times 10^{-12}\right.$ and $<6.3 \times 10^{-12}$, respectively) over the seven-day observation period (Fig. 3d, Supplementary Table 11). 
To challenge strains $\mathrm{rEc} . \beta . \mathrm{dC} .12^{\prime} . \Delta \mathrm{tY}$ and $\mathrm{rEc} . \gamma \cdot \mathrm{dC} .46^{\prime} . \Delta \mathrm{tY}$ with natural amino acids and mimic a potential HGT event, we introduced constructs containing phenylalanine or tryptophan amber suppressor tRNAs. While growth of suppressor-containing strains was equivalent to the cognate contained ancestor in permissive liquid media, severely impaired or no growth was observed in nonpermissive media (Extended Data Fig. 5). Such findings are further supported in experiments where a large $\left(\sim 10^{11}\right)$ inoculum of cells challenged on solid or in liquid (see below) nonpermissive media do not yield EMs, providing ample opportunity for natural formation of a phenylalanine amber suppressor via mutation of one of two native copies of tRNA ${ }^{\text {Phe }}$. These data support our hypothesis that synthetic auxotrophs containing higher-order TAG combinations depend on the sAA and limit growth from natural amino acids.

To interrogate the long-term stability of synthetic auxotrophs where EM formation is not limited by a colony growth environment, temporal monitoring of rEc. $\gamma \cdot \mathrm{dC} .46^{\prime} . \Delta \mathrm{tY}$ was performed on large cell populations in liquid culture (1L of LB media) for seven days with frequent $\mathrm{OD}_{600}$ measurements to track cell growth (Fig. 3e). Inoculation of $\sim 10^{11}$ cells in permissive media led to a confluent culture of contained cells within 24 hours. Inoculation of $\sim 10^{9}, \sim 10^{10}$, and $\sim 10^{11}$ cells into nonpermissive media revealed transient growth, which

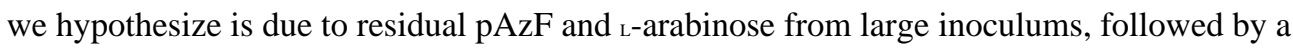
sustained decrease in cell density and growth termination. Cell survival and escape from liquid cultures was monitored by quantifying CFUs on permissive and nonpermissive solid media, respectively (Fig. 3f). Plating on permissive solid media revealed a drop in CFUs to below the limit of detection within one day from the nonpermissive flask inoculated with $\sim 10^{9}$ cells and three days from nonpermissive flasks inoculated with $\sim 10^{10}$ or $\sim 10^{11}$ cells

(Fig. 3f). No CFUs were observed from any culture plated on nonpermissive solid media. To confirm the absence of a single EM following an extended 20-day growth period (Extended Data Fig. 6), the nonpermissive and permissive cultures inoculated with $\sim 10^{11}$ cells were plated across 30 nonpermissive plates. EMs were not observed and EFs remained below the detection limit after seven days, which is comparable to the solid media results. These results demonstrate that $\mathrm{rEc} . \gamma \cdot \mathrm{dC} .46^{\prime} . \Delta \mathrm{tY}$ depends on $\mathrm{pAzF}$, maintains long-term stability of biocontainment in permissive liquid media and exhibits termination of growth in nonpermissive media.

To further investigate the dependency to sAAs, liquid growth profiles were collected for synthetic auxotrophs across SAA and L-arabinose concentration gradients. Growth of rEc. $\gamma . \mathrm{dC} .46^{\prime} . \Delta \mathrm{tY}$ was not observed below $0.002 \%$ L-arabinose and $0.5 \mathrm{mM} \mathrm{pAzF}$ (Fig. $3 \mathrm{~g}$, Extended Data Fig. 7). Growth increased in a dose-dependent manner with increasing concentrations of pAzF and $\mathrm{L}$-arabinose, where $5 \mathrm{mM} \mathrm{pAzF}$ and $0.2 \%$ L-arabinose was optimal for fitness (i.e., maximum $\mathrm{OD}_{600}$ and minimum DT). In an equivalent experiment with $\mathrm{rEc} . \beta . \mathrm{dC} .12^{\prime} . \Delta \mathrm{tY}, 1 \mathrm{mMpIF}$ and $0.2 \% \mathrm{~L}$-arabinose was optimal for fitness (Extended Data Fig. 8). Since growth was not observed in media lacking either L-arabinose or the sAA, these data further support the dependency of synthetic auxotrophs on sAAs.

To determine whether a synthetic auxotroph could be rescued by metabolic cross-feeding, we evaluated the viability of strains on diverse media types. We grew wild-type MG1655E. coli, a biotin auxotroph (EcNR2 $\left.{ }^{12}\right)$, a non-contained GRO $(\mathrm{rEc} . \gamma)$, and the pAzF synthetic 
auxotroph (rEc. $\left.\gamma . \mathrm{dC} .46^{\prime}\right)$ on solid media containing both pAzF/L-arabinose and biotin, either pAzF/L-arabinose or biotin, and on plates lacking small molecules (Fig. 4). Despite biotin auxotrophy, growth of EcNR2 on rich defined media without biotin was rescued in close proximity to wild-type $E$. coli, suggesting cross-feeding of essential metabolites (Extended Data Fig. 9). Blood agar and soil extracts without biotin or pAzF/L-arabinose supplementation supported growth of all strains except the synthetic auxotroph, which only grew on media supplemented with $\mathrm{pAzF}$ and $\mathrm{L}$-arabinose. These data suggest that synthetic auxotrophies could lead to a more viable containment strategy for clinical (e.g., blood) and environmental (e.g., soil) settings, where metabolic auxotrophies can be overcome by proximal, metabolically competent strains.

Synthetic auxotrophs utilize unnatural biochemical building blocks necessary for essential proteins with activities that cannot be complemented by naturally occurring small molecules. We have previously shown that genomic recoding interferes with HGT from viruses ${ }^{11}$ and have now extended orthogonal barriers by engineering two synthetic auxotrophs using two unique sAAs that exhibit EFs below our detection limit $\left(<6.3 \times 10^{-12}\right)$. These synthetic auxotrophs possess three essential TAGs at loci dispersed throughout the genome $(0.84,0.86$, and $2.9 \mathrm{MB}$ apart), thereby limiting the likelihood that a single HGT event could compromise containment (Extended Data Fig. 1). These orthogonal barriers can be expanded further by incorporation of additional TAG sense codons across more than three essential genes, but will likely require concurrent advances in OTS performance to maintain fitness and viability (e.g., enhanced activity and specificity of aaRS:tRNA pairs ${ }^{26}$ ). Our modular approach to biocontainment limits growth to synthetic environments containing unnatural biochemical building blocks with diverse chemistries. We anticipate that further genome recoding efforts ${ }^{11,27}$ will enable auxotrophies for multiple sAAs that could be enhanced by other synthetic components including unnatural nucleotides and extended genetic alphabets ${ }^{28-30}$. Orthogonal biological systems employing multi-level containment mechanisms are uniquely suited to provide safe GMOs for clinical, environmental, and industrial applications ${ }^{1}$.

Despite the breadth of genomic diversity found in nature, all species utilize the same biochemical foundation to sustain life. The semantic architecture of the GRO employs orthogonal translational components, establishing the basis for a synthetic molecular language that relieves limitations on natural biological functions by depending on the incorporation of sAAs with exotic chemistries. This work sets the stage for future experiments to probe the optimality of the natural genetic code and to explore the plasticity of proteins and whole organisms capable of sampling new evolutionary landscapes.

\section{Methods}

\section{Reagents}

Oligonucleotide synthesis was performed by Integrated DNA Technologies (IDT) and Keck Foundation Biotechnology Resource Laboratory at Yale University (Supplementary Table 1). Unless otherwise stated, all cultures were grown in LB medium. The following selective agents and inducers were used at the specified concentrations: ampicillin (amp, $50 \mu \mathrm{g} / \mathrm{mL}$ ), carbenicillin (carb, $50 \mu \mathrm{g} / \mathrm{mL}$ ), zeocin (zeo, $10 \mu \mathrm{g} / \mathrm{ml}$ ), spectinomycin (spec, $95 \mu \mathrm{g} / \mathrm{mL}$ ) and 
sodium dodecyl sulfate (SDS, $0.005 \% \mathrm{w} / \mathrm{v}$ ), isopropyl $\beta$-D-1-thiogalactopyranoside (IPTG, $100 \mu \mathrm{M}$ ), 5-bromo-4-chloro-3-indolyl-beta-D-galacto-pyranoside (X-Gal, $40 \mu \mathrm{g} / \mathrm{mL}$ ), and $\mathrm{L}^{-}$ arabinose (ara, $0.2 \% \mathrm{w} / \mathrm{v}$ unless otherwise indicated). sAAs were used at $1 \mathrm{mM}$ unless otherwise indicated and purchased from: PepTech (pAcF, AL624-2), BaChem (pIF, F-3075.0005), and Chem-Impex International (pAzF, 03376).

\section{Plasmids}

All tRNAs used to assess tolerance for tryptophan and phenylalanine at TAG codons were contained within the pTech plasmid backbone and driven by the lpp promoter ${ }^{31}$. Isothermal assembly ${ }^{32}$ was used to replace the chloramphenicol acetyltransferase (cat) gene with the $s h$ ble gene for resistance to zeocin.

The sup $U$ amber suppressor tRNA ${ }^{33}$ was used to assess tolerance for tryptophan and a phenylalanine amber suppressor ${ }^{31}$ was used to assess tolerance for phenylalanine. pTechsupU was graciously provided by the laboratory of Dieter Söll and supPhe was synthesized by IDT and isothermally assembled into the pTech plasmid backbone to obtain pTechsupPhe.

\section{Conversion of aminoacyl-tRNA synthetase specificity}

The pAcF OTS was integrated into the genome of the GRO linked to a counter-selectable gene tolC. Co-selection multiplex automated engineering $\left(\mathrm{CoS}-\mathrm{MAGE}^{34}\right)$ was used as described previously to introduce annotated mutations ${ }^{35}$ to the sAA binding pocket of the aaRS for specificity toward pAzF or pIF (Supplementary Table 4). Sanger sequencing was used to verify these mutations. Conversion of sAA-specificity was assessed in sequenceverified clones upon growth in the presence of sAA incorporation and episomally-expressed GFP containing an in-frame TAG codon at residue 151 within the protein product. OTSmediated suppression of this codon with the sAA (i.e., pAzF, pIF) generated a full-length fluorescent product, indicating that SAA incorporation had occurred and specificity was achieved.

\section{TAG codon incorporation into essential genes}

We applied three unique strategies to identify permissive sites in essential genes for TAG codon incorporation (Fig. 1). In our first strategy, a subset of essential genes ${ }^{36}$ were chosen for the incorporation of one or more TAG codons immediately after the start codon to encode a sAA at the amino-terminus. To explore a diverse library of incorporation targets within the E. coli proteome, in our second strategy we applied the Sorting Intolerant From Tolerant (SIFT) algorithm ${ }^{19}$ (downloaded on the Yale Biomedical High Performance Computing Cluster) to the entire panel of essential E. coli proteins ${ }^{36}$. SIFT is an algorithm that uses sequence-homology to predict the tolerance of amino acid substitutions at different indices. In our workflow, genes were first split into three categories based on wild-type expression level ${ }^{37}$, and a further four-subgroups by genomic location with the goal of targeting essential genes dispersed throughout the E. coli chromosome. Next, genes shown to be essential by multiple studies ${ }^{36}$ were passed through SIFT. For each essential gene, two high, medium, and low tolerance sites were targeted for TAG incorporation by MAGE. By 
this approach, we were able to sample diverse residue types in proteins with varying wildtype expression levels.

In our third strategy, using the conserved domain database ${ }^{13}$, we searched within all annotated essential proteins for tyrosine, tryptophan and phenylalanine residues predicted to participate in 1) essential enzymatic reactions or 2) protein-protein interactions (e.g., dimerization). To minimize the probability that the added functionality of the sAA would perturb protein function, we targeted sites that were observed to occur as tyrosine or tryptophan in different homologs.

GROs containing an OTS integrated into the chromosome were grown to mid-log phase in liquid permissive LB and four cycles of MAGE were performed per pool of mutagenic oligos (oligo concentration $\leq 15 \mu \mathrm{M}$ ) as described previously ${ }^{12,38}$. To isolate synthetic auxotrophs, mutagenized cultures were plated on solid media and replica plated onto nonpermissive media. To identify TAG incorporation loci, MASC-PCR was used to interrogate pools of up to eleven targeted loci as previously described ${ }^{38}$, followed by verification using Sanger sequencing.

\section{Genotyping}

Sanger sequencing was performed by the Keck DNA Sequencing Facility at Yale or by GENEWIZ, Inc. Genomic DNA for whole genome sequencing was prepared using a Qiagen Genomic DNA purification kit. Illumina libraries were prepared by the Yale Center for Genomic Analysis or the Dana Farber Cancer Institute. Illumina HiSeq or MiSeq sequencing systems were used for whole genome sequencing to generate 50 or 150 bp paired-end reads, respectively.

Whole genome sequencing was used to analyze three EMs per background. In all cases, the direct ancestor to the EM was also analyzed. SNPs in EMs were identified relative to the reference genome Escherichia coli C321. $\mathrm{A}$ (CP006698.1, GI:54981157) using a previously described ${ }^{11}$ software pipeline. SNPs listed in Supplementary Table 8 and Supplementary Table 10 were called by Freebayes in EMs.

\section{Strains}

All GROs used in this study are derived from C321. $\Delta$ A (CP006698.1, GI:54981157) ${ }^{38}$ which lacks all TAG codons and release factor 1. This strain is derived from strain EcNR2 ( $\Delta m u t S: c a t \Delta(y b h B$-bioAB):[cI857A(cro-ea59):tetR-bla]), modified from E. coli K-12 substr. $M G 1655$. In all synthetic auxotrophs, the $M$. jannaschii-derived OTS was genomically integrated into the GRO fused to the counter-selectable gene tolC. The OTS consists of an Larabinose-inducible aaRS driven by the $\operatorname{araBAD}$ promoter, and a constitutively expressed cognate amber-decoding tRNA driven by the proK promoter. All genome modifications that required incorporation of dsDNA (e.g., modifications to the mutS gene, incorporation of antibiotic selectable markers) were performed via $\lambda$-Red recombination ${ }^{39}$. 


\section{Nomenclature of genomically recoded organisms and synthetic auxotrophs}

Number of essential TAG codons-To succinctly name strains, we have introduced a new one-letter amino acid code for sAAs using Greek lettering $(\mathrm{pAcF}=\alpha, \mathrm{pIF}=\beta$, and $\mathrm{pAzF}=\gamma$ ). Non-contained GROs lacking essential TAG codons are named according to the one letter sAA code for the specific OTS present in the organism. For example, a $\Delta$ TAG GRO with a genomically integrated pAcF OTS is rEc.a.

Biocontained GROs containing essential TAG codons are named according to two conventions based on the number of essential TAG codons in the auxotroph: 1) Strains with one essential TAG are named by the essential protein containing the sAA and the position and identity of the residue substituted therein (e.g., a strain containing $\mathrm{pAcF}$ at residue 113 in DnaX is DnaX.Y113a); 2) Strains containing more than one essential TAG are named using the one letter sAA code for which the organism is auxotrophic. This is followed by a dependency code, $d$, indicating the presence of two $(\mathrm{dB})$, three $(\mathrm{dC})$ or four $(\mathrm{dD})$ essential TAG codons, and then by a TAG combination number that uniquely identifies the specific combination of TAGs in the strain. Combinations are numbered from one through 46 and are listed in Supplementary Table 6.

Mismatch repair-The presence of a prime (') following the TAG combination number indicates that $m u t S$ has been restored at its native locus, imparting functional mismatch repair to the organism.

tRNA redundancy-Following the TAG combination number, $\Delta \mathrm{t}$ indicates the amino acid for which tRNA redundancy has been eliminated and is followed by the relevant amino acid (e.g., a strain in which two of three total tyrosine tRNAs were deleted is $\Delta \mathrm{tY}$ ).

Escape mutant identity-At least three EMs were characterized per strain background that permitted an escape mutant. An EM is designated by a number following the letter "E" (e.g., E1).

The summary of synthetic auxotrophs generated in this study illustrated in Extended Data Fig. 1 was constructed using the $\operatorname{Circos}{ }^{40}$ software.

\section{Fitness analysis}

Strains were grown at $34^{\circ} \mathrm{C}$ in flat-bottomed 96-well plates containing $150 \mu \mathrm{L}$ of $\mathrm{LB}$ medium permissive for sAA incorporation, unless otherwise indicated. Strains were washed twice with sterile $\mathrm{dH}_{2} \mathrm{O}$ prior to assessing growth in nonpermissive media. Kinetic growth $\left(\mathrm{OD}_{600}\right)$ was monitored on a BioTek plate reader at ten-minute intervals in triplicate. Raw $\mathrm{OD}_{600}$ data from the plate reader were normalized to standard absorbance $\left(\mathrm{OD}_{600}\right.$ at $1 \mathrm{~cm}$ path length) values using an empirically derived calibration curve $(y=1.9704 \times-0.1183$, where $y=\mathrm{OD}_{600}$ at $1 \mathrm{~cm}$ path length and $\mathrm{x}=\mathrm{OD}_{600}$ from plate reader; $\mathrm{R}^{2}=0.998$ ). DTs were calculated in MATLAB using custom code. Reported values are the average between three technical replicates where error bars represent \pm s.d. All reported results repeated at least three times in independent experiments. Maximum $\mathrm{OD}_{600}$ values were obtained after 
24 hours of growth and represent the average of three technical replicates. Reported results repeated at least three times in independent experiments.

\section{Mass spectrometry}

Histidine-tagged proteins were purified on NiNTA resin (Qiagen). Resolution of purity was assessed via SDS-PAGE. In gel digestion was performed similarly to previously described methods ${ }^{41}$. Proteins were stained and imaged within the gel using Coomassie Blue (R-250). A band corresponding to the molecular weight of DnaX was excised. Gel slices were processed into $1 \mathrm{~mm}$ cubes, washed in 1:1 (v/v) $50 \% \mathrm{CH}_{3} \mathrm{CN} / 50 \mathrm{mM} \mathrm{NH}_{4} \mathrm{HCO}_{3}$, and then washed in 1:1 (v/v) $50 \% \mathrm{CH}_{3} \mathrm{CN} / 10 \mathrm{mM} \mathrm{NH}_{4} \mathrm{CO}_{3} .13 .33 \mathrm{ng} / \mu \mathrm{l}$ trypsin solution in 9:1 (v/v) $50 \mathrm{mM} \mathrm{NH}_{4} \mathrm{CO}_{3} / 50 \% \mathrm{CH}_{3} \mathrm{CN}$ was added and samples were incubated overnight at $37^{\circ} \mathrm{C}$. Peptides were extracted with 1:2 (v/v) 5\% formic acid/50\% $\mathrm{CH}_{3} \mathrm{CN}$ and dried. Peptides were desalted by reconstitution in 3:8 (v/v) $70 \%$ formic acid/ $/ .1 \%$ TFA, followed by loading onto a custom-made stage tip $(2 \times 1.06 \mathrm{~mm}$ punches of Empore $\mathrm{C} 18$ extraction disks [3 M] in a $200 \mu$ l pipette tip) ${ }^{42}$ activated with $80 \% \mathrm{CH}_{3} \mathrm{CN}$ and $0.1 \%$ TFA. Tips were washed twice with $0.1 \%$ TFA and peptides eluted with $80 \% \mathrm{CH}_{3} \mathrm{CN}$ and $0.1 \%$ TFA. Peptides were dried and reconstituted for LC/MS/MS analysis. Capillary LC/MS/MS was carried out using an LTQ Orbitrap Velos (Thermo Scientific) with a nanoAcquity uHPLC (Waters) system as described previously ${ }^{43}$. The data were processed as described previously ${ }^{11}$. MASCOT scores were above the identity or extensive homology threshold and representative spectra are illustrated in Fig. 2c.

\section{Quantitative assessment of amino acid tolerance}

The following workflow was performed to assess the tolerance for natural amino acid substitution at residues chosen for sAA incorporation. Strains were grown to mid-log phase in $1 \mathrm{~mL}$ of permissive $\mathrm{LB}$, and MAGE was performed as described ${ }^{12}$ with modifications described here. Post induction of $\lambda$-Red proteins, cells were transferred to individual wells of a 96-well, V-bottomed plate, and washed twice at $4{ }^{\circ} \mathrm{C}$ with sterile $\mathrm{dH}_{2} \mathrm{O}$. Cells were resuspended in $50 \mu \mathrm{l}$ of water or $1 \mu \mathrm{M}$ mutagenic ssDNA to convert a single in-frame essential TAG codon to one of 20 sense codons, and electroporated in a 96-well plate. Cells were electroporated using the Harvard BTX electroporation system $(2.4 \mathrm{kV}, 750 \Omega, 25 \mu \mathrm{F})$. Electroporated cells were recovered in $1.5 \mathrm{~mL}$ of fresh permissive media in a 96-well plate for four hours at $34^{\circ} \mathrm{C}$ shaking. Cells were pelleted, washed twice with sterile $\mathrm{dH}_{2} \mathrm{O}$, and resuspended in $200 \mu \mathrm{L}$ of $1 \times$ PBS. Serial dilutions were made in $1 \times \mathrm{PBS}$ and $50 \mu \mathrm{L}$ each of non-diluted and 100-fold diluted samples were plated on solid nonpermissive LB media. 50 $\mu \mathrm{L}$ each of higher dilutions were plated on permissive solid media and all plates were incubated for 20 hours at $34^{\circ} \mathrm{C}$.

CFUs counted on nonpermissive media were expressed as a ratio of total CFUs on permissive media. Since the frequency of MAGE-mediated recombination $(\sim 0.3)^{12}$ exceeds the EFs of these background strains $\left(\leq 10^{-5}\right)$, we directly correlated these ratios to amino acid tolerance. MATLAB was used to calculate the $\log _{10}$ of this ratio. Where no CFUs were observed on nonpermissive media, indicative of a highly intolerant substitution, a ratio could not be calculated and these values were defaulted to NaN within MATLAB. A heat map was 
used to compare representative data for one experiment, where blue indicates a tolerated substitution and yellow, a non-tolerated substitution.

Twenty-one separate MAGE experiments were performed as described above for each strain, per essential genomic TAG, to assess tolerance for each of the 20 natural amino acids at each TAG site, plus a negative control (water). Strains with one TAG codon (SecY.Y122 $\beta$, DnaX.Y113 $\beta$, LspA.Y54 $\beta$, DnaA.W6 $\gamma$, SerS.F213 $\gamma$, and MurG.F243 $\gamma$ ) were assessed across 21 (including the negative control) experiments per strain, strains with two TAG codons (rEc.P.dB.9) were assessed across 42 (including two negative control)

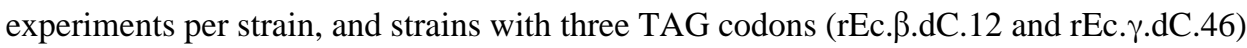
were assessed across 63 (including three negative control) experiments per strain. Reported results repeated at least three times in independent experiments.

\section{Escape assays}

Strains were grown in triplicate to late-log phase in $2 \mathrm{~mL}$ of permissive $\mathrm{LB}$, pelleted, washed twice with sterile $\mathrm{dH}_{2} \mathrm{O}$, and re-suspended in $200 \mu \mathrm{L} 1 \times$ PBS. To obtain total and EM CFUs, serial dilutions were made and equal volumes were plated on permissive and nonpermissive solid media plates $(100 \times 15 \mathrm{~mm})$. Plates were incubated at $34^{\circ} \mathrm{C}$ and $\mathrm{EF}$ was calculated as the total number of EM CFUs observed per total cells plated. Reported EFs are means of three technical replicates where error bars represent \pm s.d. To isolate EMs in strains with lower EFs, $\sim 10^{10}-10^{11}$ cells were plated and the resulting EF from a representative escape assay is reported. When EMs were not detected upon plating $\sim 10^{10}-10^{11}$ cells, the $\mathrm{EF}$ is described to be below the limit of detection and reported as less than a frequency of one over the total number of cells plated. In all cases, reported results repeated at least three times in independent experiments. Where temporal monitoring of EFs on solid media is reported (Fig. 3d), representative escape assays are plotted and results repeated at least three times in independent experiments.

\section{Liquid escape assays}

Long term liquid growth was assessed for two strain backgrounds: the pAzF-dependent strain, $\mathrm{rEc} . \gamma \cdot \mathrm{dC} .46^{\prime} . \Delta \mathrm{tY}$ and its non-contained ancestor, $\mathrm{rEc} . \gamma$. Growth of rEc. $\gamma \cdot \mathrm{dC} .46^{\prime} . \Delta \mathrm{tY}$ was separately assessed in permissive (+sAA/ $+\mathrm{L}-$ arabinose) and nonpermissive (-sAA/-Larabinose) media and growth of $\mathrm{rEc} . \gamma$ was assessed in nonpermissive media. In all cases, flasks contained carbenicillin to prevent contamination.

Strains were grown in $100 \mathrm{ml}$ of LB media overnight. Cultures were then pelleted and washed twice with the same volume of sterile $\mathrm{dH}_{2} \mathrm{O}$. Washed pellets were re-suspended in LB media plus or minus small molecules and this slurry was then added to shake flasks containing $1 \mathrm{~L}$ of LB media plus or minus small molecules, at time zero. At this first time point, a $1 \mathrm{ml}$ sample was obtained from each flask, from which the $\mathrm{OD}_{600}$ was measured and $50 \mu \mathrm{l}$ was plated on both permissive (+sAA/ $+\mathrm{L}-$ arabinose/+carbenicillin) and nonpermissive (-sAA/-L-arabinose/+carbenicillin) solid LB media, in three technical replicates. Average $\mathrm{CFU}$ counts are reported and error bars represent \pm s.d. In all cases, CFUs on solid media were counted after 24 hours of incubation at $34^{\circ} \mathrm{C}$. Time points were collected in this manner for all subsequent time points for the following 20 days. 
After 20 days of growth in liquid media, the two $1 \mathrm{~L}$ cultures of $\mathrm{rEc} . \gamma \cdot \mathrm{dC} .46^{\prime} . \Delta \mathrm{tY}$ grown in nonpermissive and permissive media were interrogated for the presence of a single EM. The entire culture was pelleted, re-suspended in $7 \mathrm{ml}$ of $1 \times \mathrm{PBS}$, and plated across 30 large nonpermissive solid media plates that were subsequently monitored for CFU formation over the following seven-day period. All reported results repeated at least three times in independent experiments.

\section{Environmental challenges}

Wild-type E. coli K-12 substr. MG1655, and additional E. coli strains EcNR2 ${ }^{12}$, rEc. $\gamma . \mathrm{dC}$. $46^{\prime}$ (a pAzF auxotroph), and $\mathrm{rEc} . \gamma$ (non-contained ancestor to $\mathrm{rEc} . \gamma \cdot \mathrm{dC} .46^{\prime}$ ) were grown to mid-log phase in $1 \mathrm{~mL}$ of LB media supplemented with small molecules, where necessary. Cultures were washed three times with sterile $\mathrm{dH}_{2} \mathrm{O}$ and re-suspended in $1 \mathrm{~mL} 1 \times$ PBS. A total of 16, two-fold serial dilutions were made and spotted on the following solid media types: LB, EZ Rich Defined ${ }^{44}$ (with modifications by Teknova) containing 100× carbon source: 40\% glycerol, Blood Agar (Teknova), or Soil Extract Agar (HiMedia). Prior to spotting, plates were topically supplemented with pAzF, L-arabinose, and/or biotin, and dried for at least one hour. Spotted plates were incubated for one day at $34^{\circ} \mathrm{C}$ and photographed in a Gel Doc XR+ running ImageLab v4.0.1 (BioRad).

\section{Selectable markers used in this study $>\operatorname{cat}(1,015 \mathrm{bp})$}

cetgtgacggaagatcacttcgcagaataaataaatcctggtgtccetgttgataccgggaagccetgggccaacttttggcgaaaat gagacgttgatcggcacgtaagaggttccaactttcaccataatgaaataagatcactaccgggcgtattttttgagttgtcgagattttc aggagctaaggaagctaaaatggagaaaaaaatcactggatataccaccgttgatatatcccaatggcatcgtaaagaacattttgag gcatttcagtcagttgctcaatgtacctataaccagaccgttcagctggatattacggcctttttaaagaccgtaaagaaaaataagcac aagttttatccggcetttattcacattcttgcccgcctgatgaatgctcatccggaattacgtatggcaatgaaagacggtgagctggtga tatgggatagtgttcaccettgttacaccgttttccatgagcaaactgaaacgttttcatcgctctggagtgaataccacgacgatttccg gcagtttctacacatatattcgcaagatgtggcgtgttacggtgaaaacctggcctatttccctaaagggtttattgagaatatgtttttcgt ctcagccaatccctgggtgagtttcaccagttttgatttaaacgtggccaatatggacaacttcttcgcccccgttttcaccatgggcaaa tattatacgcaaggcgacaaggtgctgatgccgctggcgattcaggttcatcatgccgtttgtgatggcttccatgtcggcagaatgctt aatgaattacaacagtactgcgatgagtggcagggcggggcgtaattttttaaggcagttattggtgccttaaacgcetggttgctac gcctgaataagtgataataagcggatgaatggcagaaattcgaaagcaaattcgacccggtcgtcggttcagggcagggtcgttaaa tagccgettatgtctattgetggtt

\section{$>\operatorname{kanR}(1,165 \mathrm{bp})$}

cctgtgacggaagatcacttcgcagaataaataaatcctggtgtccetgttgataccgggaagccetgggccaactttggcgaaaat gagacgttgatcggcacgtaagaggttccaactttcaccataatgaaataagatcactaccgggcgtattttttgagttgtcgagattttc aggagctaaggaagctaaaatgagccatattcaacgggaaacgtcgaggccgcgattaaattccaacatggatgetgatttatatgg gtataaatgggctcgcgataatgtcgggcaatcaggtgcgacaatctatcgcttgtatgggaagcccgatgcgccagagttgtttctg aaacatggcaaaggtagcgttgccaatgatgttacagatgagatggtcagactaaactggctgacggaatttatgectcttccgaccat caagcattttatccgtactcctgatgatgcatggttactcaccactgcgatccccggaaaaacagcattccaggtattagaagaatatcc tgattcaggtgaaaatattgttgatgcgctggcagtgttcctgcgecggttgcattcgattcctgtttgtaattgtccttttaacagcgatcg cgtattcgtctcgctcaggcgcaatcacgaatgaataacggtttggttgatgcgagtgattttgatgacgagcgtaatggctggcctgt tgaacaagtctggaaagaaatgcataaacttttgccattctcaccggattcagtcgtcactcatggtgatttctcacttgataaccttattttt 
gacgaggggaaattaataggttgtattgatgttggacgagtcggaatcgcagaccgataccaggatcttgccatcctatggaactgcc tcggtgagttttctccttcattacagaaacggctttttcaaaaatatggtattgataatcctgatatgaataaattgcagtttcatttgatgctc gatgagtttttctaatttttttaaggcagttattggtgcccttaaacgcctggttgctacgcctgaataagtgataataagcggatgaatgg cagaaattcgaaagcaaattcgacccggtcgtcggttcagggcagggtcgttaaatagccgettatgtctattgctggtt

\section{$>\operatorname{spec}^{R}(1,201 \mathrm{bp})$}

cagccaggacagaaatgcctcgacttcgetgctgcccaaggttgccgggtgacgcacaccgtggaaacggatgaaggcacgaac ccagtggacataagcetgttcggttcgtaagctgtaatgcaagtagcgtatgcgctcacgcaactggtccagaacettgaccgaacg cagcggtggtaacggcgcagtggcggttttcatggcttgttatgactgttttttggggtacagtctatgcetcgggcatccaagcagca agcgcgttacgccgtgggtcgatgtttgatgttatggagcagcaacgatgttacgcagcagggcagtcgccctaaaacaaagttaaa catcatgagggaagcggtgatcgecgaagtatcgactcaactatcagaggtagttggcgtcatcgagcgecatctcgaaccgacgtt gctggccgtacatttgtacggctccgcagtggatggcggcctgaagccacacagtgatattgatttgctggttacggtgaccgtaagg cttgatgaaacaacgcggcgagctttgatcaacgacctttggaaacttcggcttccctggagagagcgagattctccgcgctgtag aagtcaccattgttgtgcacgacgacatcattccgtggcgttatccagctaagcgcgaactgcaatttggagaatggcagcgcaatga cattcttgcaggtatcttcgagccagccacgatcgacattgatctggctatcttgctgacaaaagcaagagaacatagcgttgccttggt aggtccagcggcggaggaactctttgatccggttcctgaacaggatctatttgaggcgctaaatgaaaccttaacgctatggaactcg ccgcccgactgggctggcgatgagcgaaatgtagtgcttacgttgtcccgcatttggtacagcgcagtaaccggcaaaatcgcgec gaaggatgtcgctgccgactgggcaatggagcgcctgccggccagtatcagccegtcatacttgaagctagacaggcttatcttgg acaagaagaagatcgcttggcctcgegcgcagatcagttggaagaatttgtccactacgtgaaaggcgagatcaccaaggtagtcg gcaaataaagctttactgagctaataacaggactgctggtaatcgcaggccttttatttctgca

\section{$>\operatorname{tol} C(1,746 \mathrm{bp})$}

ttgaggcacattaacgecetatggcacgtaacgccaaccttttgcggtagcggcttctgctagaatccgcaataattttacagtttgatc gcgctaaatactgcttcaccacaaggaatgcaaatgaagaaattgctcccattcttatcggcetgagcetttctgggttcagttcgttga gccaggccgagaacctgatgcaagtttatcagcaagcacgccttagtaacccggaattgcgtaagtctgccgccgatcgtgatgctg cetttgaaaaaattaatgaagcgcgcagtccattactgccacagctaggtttaggtgcagattacacctatagcaacggctaccgcga cgcgaacggcatcaactctaacgcgaccagtgcgtccttgcagttaactcaatccatttttgatatgtcgaaatggcgtgcgttaacgct gcaggaaaaagcagcagggattcaggacgtcacgtatcagaccgatcagcaaaccttgatcctcaacaccgcgaccgcttattca acgtgttgaatgctattgacgttctttcctatacacaggcacaaaaagaagcgatctaccgtcaattagatcaaaccacccaacgtttta acgtgggcetggtagcgatcaccgacgtgcagaacgccegcgcacagtacgataccgtgctggcgaacgaagtgaccgcacgta ataaccttgataacgcggtagagcagctgcgccagatcaccggtaactactatccggaactggctgcgctgaatgtcgaaaacttaa aaccgacaaaccacagccggttaacgcgctgctgaaagaagccgaaaaacgcaacctgtcgctgttacaggcacgcttgagccag gacctggcgcgcgagcaaattcgccaggcgcaggatggtcacttaccgactctggatttaacggettctaccgggatttctgacacct cttatagcggttcgaaaacccgtggtgccgctggtacccagtatgacgatagcaatatgggccagaacaaagttggcctgagcttctc gctgccgatttatcagggcggaatggttaactcgcaggtgaaacaggcacagtacaactttgtcggtgccagcgagcaactggaaa gtgccatcgtagcgtcgtgcagaccgtgcgttcctccttcaacaacattaatgcatctatcagtagcattaacgcctacaaacaagcc gtagtttccgctcaaagctcattagacgcgatggaagcgggctactcggtcggtacgcgtaccattgttgatgtgttggatgcgacca ccacgttgtacaacgccaagcaagagctggcgaatgcgcgttataactacctgattaatcagctgaatattaagtcagctctgggtac gttgaacgagcaggatctgctggcactgaacaatgcgctgagcaaaccggtttccactaatccggaaaacgttgcaccgcaaacgc cggaacagaatgctattgctgatggttatgcgectgatagcceggcaccagtcgttcagcaaacatccgcacgcactaccaccagta acggtcataacctttccgtaactgatgacgacgacggggaagcttaattagctgatctagaggcatcaaataaaacgaaaggctca gtcgaaagactgggectttcgttttatctgttgtttgtcggtgaacgetctcctgagtaggacaaatccgccgecctaga

Nature. Author manuscript; available in PMC 2015 October 01. 
$>z e o^{R}(762 \mathrm{bp})$

ggtgttgacaattaatcatcggcatagtatatcggcatagtataatacgacaaggtgaggaactaaaccatggccaagttgaccagtg ccgttccggtgctcaccgcgcgcgacgtcgccggagcggtcgagttctggaccgaccggctcgggttctcccgggacttcgtgga ggacgacttcgccggtgtggtccgggacgacgtgaccetgttcatcagcgcggtccaggaccaggtggtgccggacaacaccetg gcctgggtgtgggtgcgcggcctggacgagctgtacgccgagtggtcggaggtcgtgtccacgaacttccgggacgcctccggg ccggccatgaccgagatcggcgagcagccgtgggggegggagttcgccetgcgcgacccggccggcaactgcgtgcacttcgt ggccgaggagcaggactgacacgtccgacggeggcccacgggtcccaggcctcggagatccgtccccttttcctttgtcgatatc atgtaattagttatgtcacgcttacattcacgccetcccccacatccgctctaaccgaaaaggaaggagttagacaacctgaagtcta ggtccctatttattttttatagttatgttagtattaagaacgttatttatatttcaaattttctttttttctgtacagacgcgtgtacgcatgtaac attatactgaaaaccttgcttgagaaggttttgggacgctcgaaggctttaatttgcaagct

\section{Extended Data}

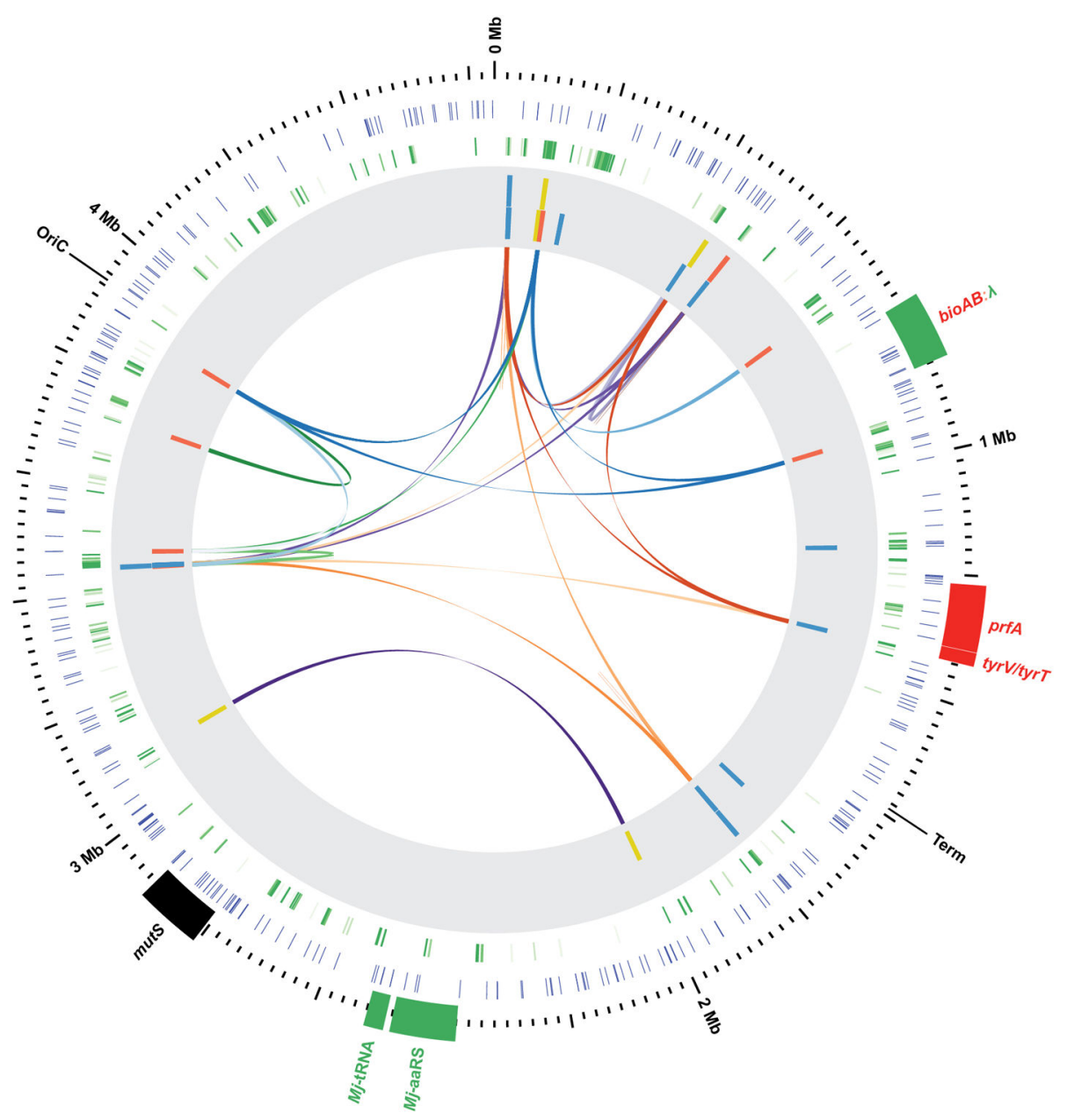

Extended Data Figure 1. Comprehensive map of synthetic auxotrophs

Circos plot summarizing synthetic auxotrophs generated in this study. Red and green genes reflect knockouts and insertions, respectively. Outermost ticks indicate genomic location, inner blue ticks indicate locations where TAG codons were converted to TAA in the GRO, and green ticks reflect locations of 303 E. coli essential genes. The shaded grey inner circle contains essential TAG loci in synthetic auxotrophs, where yellow ticks represent amino- 
terminal insertions, blue ticks represent tolerant substitutions, and red ticks represent functional site substitutions. Innermost links represent unique combinations of TAGs in higher-order synthetic auxotrophs. Links of a single color correspond to a single strain.

a
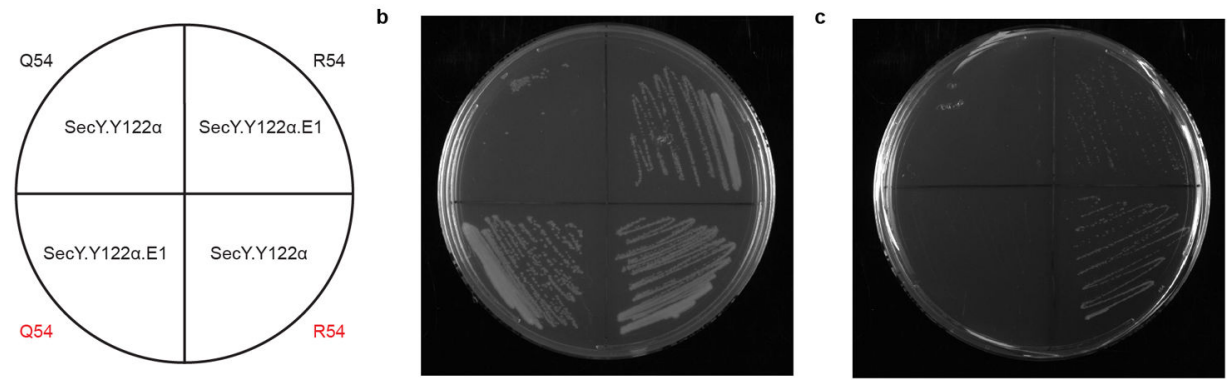

Extended Data Figure 2. rpsD.Q54R is sufficient for loss of pAcF-dependence in SecY.Y122a a, Plate map with genotypes of strains shown in b and c. Top half of plate: SecY.Y122a.E1 (upper right quadrant) contains the rpsD.Q54R mutation and is an EM of pAcF-auxotroph, SecY.Y122a (upper left quadrant). Lower half of plate: The rpsD.Q54R mutation was introduced into SecY.Y122a (lower right quadrant), resulting in a loss of pAcF-dependence, and reverted to wild type in SecY.Y122a.E1 (lower left quadrant), restoring pAcFdependence. The amino acid present at residue 54 within RpsD is indicated at the perimeter of the plate, where red signifies that the given mutation was introduced into the genotype by MAGE to demonstrate the causal mechanism of escape. $\mathbf{b}$, Growth on solid permissive media demonstrates growth of all strains. c, Growth on solid nonpermissive media. Introduction of the rpsD.Q54R mutation into the synthetic auxotroph SecY.Y122a results in loss of containment (lower right quadrant). Reverting the mutation to wild type in SecY.Y122a.E1 results in restoration of containment (lower left quadrant). Together, these data demonstrate that the rpsD.Q54R mutation is sufficient for loss of pAcF-dependence in SecY.Y122a. 


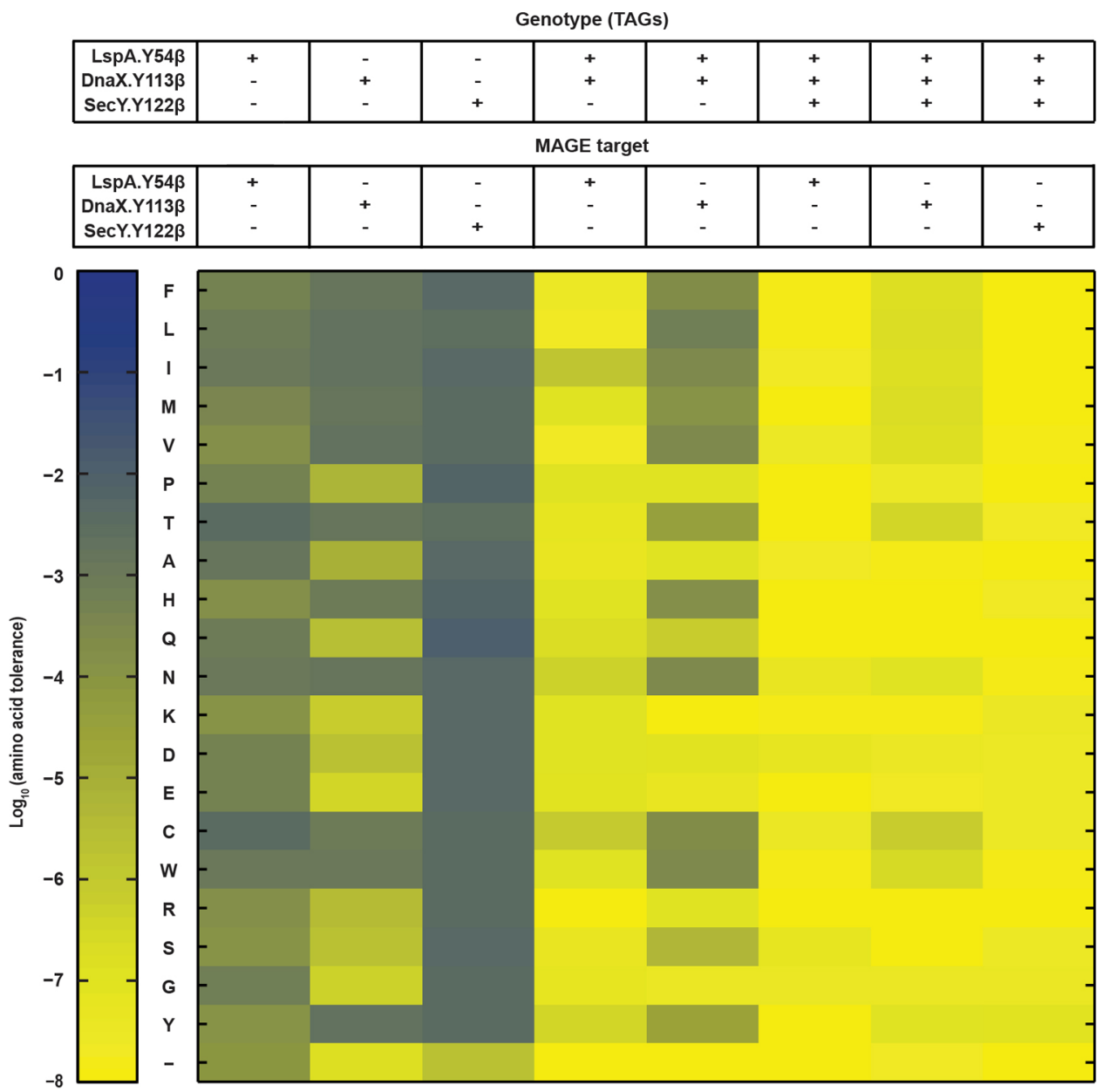

Extended Data Figure 3. Quantitative assessment of amino acid tolerance in higher-order pIF auxotrophs

Representative assay surveying tolerance of one of three essential TAG loci to the twenty amino acids in different synthetic auxotrophs and expressed as $\log _{10}$ of total cell survival. The + symbol indicates the presence of a TAG codon at the specified locus in the background strain and - indicates the wild type codon. Blue and yellow indicate high and low tolerance to substitution, respectively. Substitutions DnaX.Y113W and SecY.Y122Q are tolerated but yielded a lower percentage of survival on nonpermissive media in a background with two TAGs, an effect that was pronounced in a background with three TAGs. While DnaX.Y113, SecY.Y122 and LspA.Y54 are permissive for most natural amino acids, strains with more than one of these essential TAGs are less prone to survive in the event that any one TAG is compromised. SecY.Y122Q and DnaX.Y113W were tolerated substitutions also observed in real EMs of these strains (Supplementary Table 7). Refer to the methods for a complete description of this experiment. 


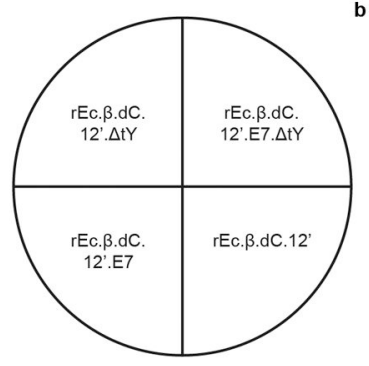

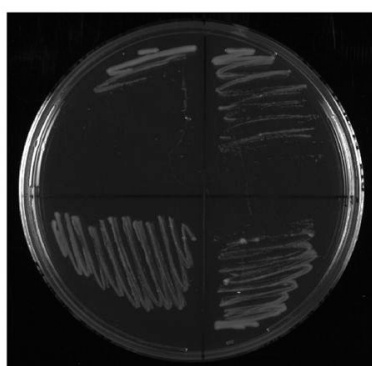

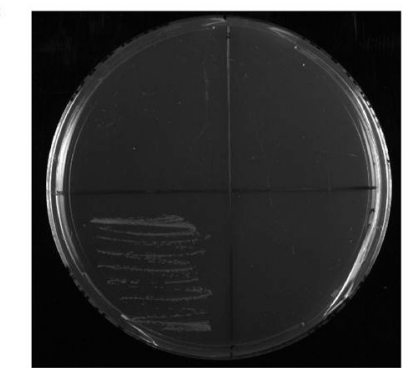

Extended Data Figure 4. Deletion of $t y r T$ and $t y r V$ restores pIF-dependence and fitness of rEc.ß.dC.12'.E7

a, Plate map with genotypes of strains in parts b and c. rEc. $\beta . d C .12^{\prime}$.E7 is an EM of its sAAdependent ancestor (rEc. $\left.\beta . \mathrm{dC} .12^{\prime}\right)$ and contains a tyrT ochre suppressor mutation ( $\operatorname{supC}$ ).

The fitness of rEc. $\beta . \mathrm{dC} .12^{\prime}$.E7 in permissive media is impaired relative to rEc. $\beta . \mathrm{dC} .12^{\prime}$, with DTs of $91.74( \pm 1.49)$ and $61.81( \pm 0.65)$ minutes, respectively. Tyrosine tRNA redundancy was eliminated $(\Delta \mathrm{tY})$ in both strains by $\lambda$-Red mediated replacement of $t y r T$ and $t y r V$ with chloramphenicol acetyltransferase (cat), rendering the resulting strains (rEc. $\beta . d C .12^{\prime} . \Delta \mathrm{tY}$ and $\mathrm{rEc} . \beta . \mathrm{dC} .12^{\prime}$.E7. $\Delta \mathrm{tY}$ ) dependent on $t y r U$ for tyrosine incorporation during normal protein synthesis. Elimination of tyrosine redundancy reduced the EF of rEc. $\beta . d C .12^{\prime}$ from $2.17 \times 10^{-9}$ (Fig. 2 e) to $<4.85 \times 10^{-12}$ (no EMs were observed upon plating $2.06 \times 10^{11}$ cells) and restored pIF-dependence in rEc. $\beta . d C .12^{\prime}$.E7 to $<4.73 \times 10^{-12}$ (no EMs were observed upon plating $2.12 \times 10^{11}$ cells). EMs were not detected for either strain up to seven days after plating on nonpermissive media (Fig. $3 \mathrm{~d}$ and Supplementary Table 11). Tyrosine tRNA deletion also restored the fitness of the EM to approximately that of its sAA-dependent ancestor (60.66 \pm 0.12 minutes). Taken together, these results establish $t y r T$ as the causal mechanism of escape in rEc. $\beta . d C .12^{\prime}$.E7. b, Growth on solid permissive LB media. c, Growth on solid nonpermissive LB media. All reported DTs are averages, where $n=3$ technical replicates, and error bars are \pm s.d. Refer to the methods for a complete description of EFs. 


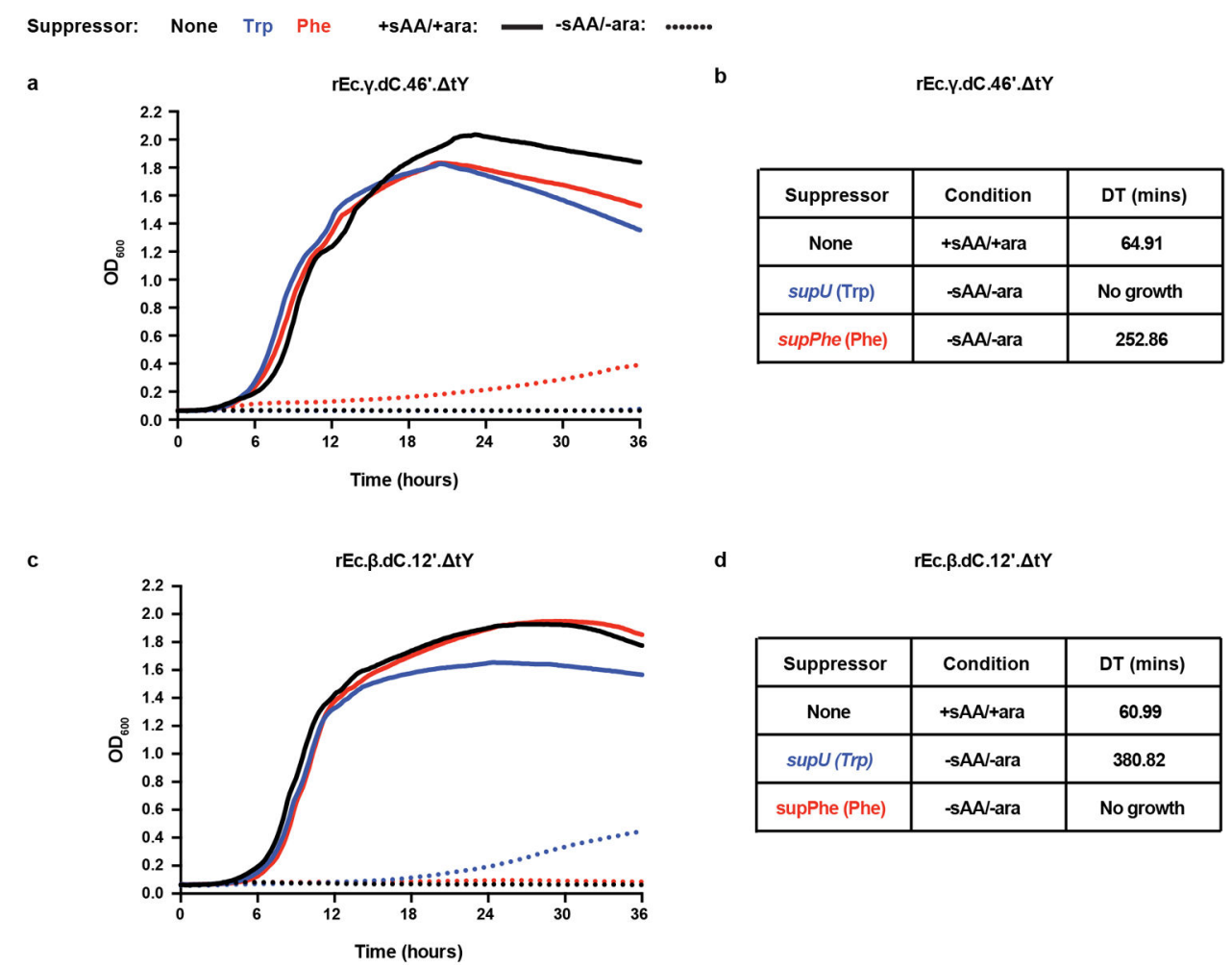

Extended Data Figure 5. Growth profiles of strains expressing phenylalanine or tryptophan amber suppressor tRNAs

Growth was assessed for $\mathrm{rEc} . \gamma \cdot \mathrm{dC} .46^{\prime} . \Delta \mathrm{tY}$ and $\mathrm{rEc} . \beta . \mathrm{dC} .12^{\prime} . \Delta \mathrm{tY}$ in the presence of amber suppression by either pTech-supU (blue), pTech-supPhe (red), or in the absence of plasmidbased amber suppression (black). Cells were washed twice with $\mathrm{dH}_{2} \mathrm{O}$ and re-suspended in the same volume of $1 \times$ PBS. Washed cells were normalized by $\mathrm{OD}_{600}$ to inoculate roughly equal numbers of cells per well. Growth profiles are shown for $\mathbf{a}, \mathbf{b}, \mathrm{rEc} . \gamma \cdot \mathrm{dC} .46^{\prime} . \Delta \mathrm{tY}$ and $\mathbf{c}$, d, $\mathrm{rEc} . \beta . \mathrm{dC} .12^{\prime} . \Delta \mathrm{tY}$ in permissive (+sAA/+ ${ }_{\mathrm{L}}$-arabinose, solid lines) and nonpermissive ($\mathrm{SAA} / \mathrm{-L}$-arabinose, dashed lines) LB liquid media. DTs are shown for the ancestral strain (black) in permissive media and suppressor-containing strains (red and blue) in nonpermissive media where growth was observed. Plasmid containing strains were always grown in the presence of zeocin for plasmid maintenance. Growth was never observed for the contained ancestors in nonpermissive media (black, dashed lines). In the presence of tryptophan suppression, growth of rEc. $\gamma \cdot \mathrm{dC} .46^{\prime} . \Delta \mathrm{tY}$ was not observed and growth of rEc. $\beta . d C .12^{\prime} . \Delta \mathrm{tY}$ was severely impaired (380 minute DT) with a 6.24-fold increase in DT relative to the contained ancestor grown in permissive media. In the presence of phenylalanine suppression, growth of rEc. $\beta . \mathrm{dC} .12^{\prime} . \Delta \mathrm{tY}$ was not observed and growth of rEc. $\gamma . \mathrm{dC} .46^{\prime} . \Delta \mathrm{tY}$ was severely impaired (252 minute DT) with a 3.90 -fold increase in DT relative to the contained ancestor grown in permissive media. Representative growth profiles and DTs are reported. These results repeated at least three times in individual experiments. 


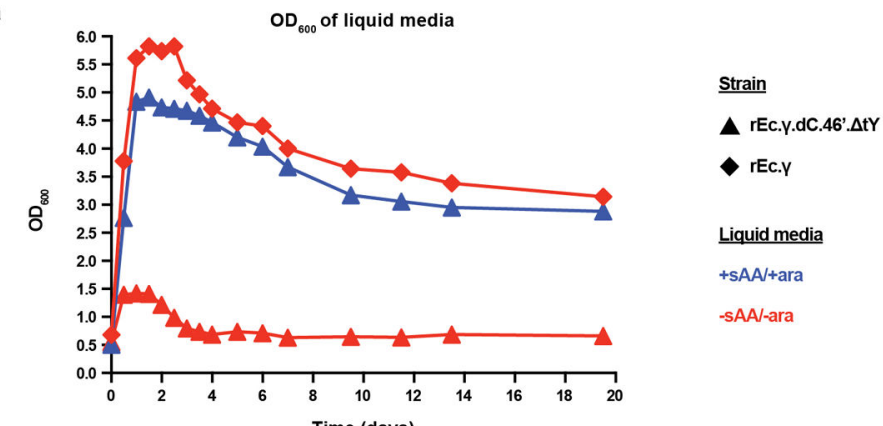

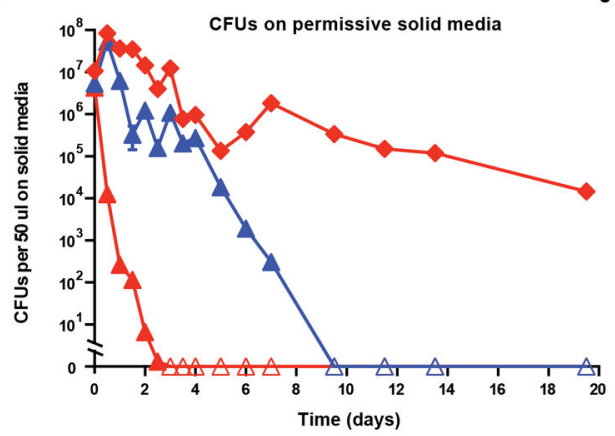

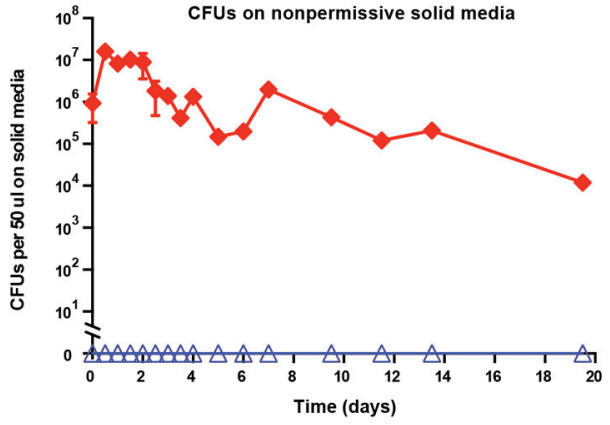

Extended Data Figure 6. Long term growth of rEc. $\gamma . d C .466^{\prime} . \Delta t Y$ in liquid LB media relative to rEc. $\gamma$

Approximately $10^{11}$ cells of strain $\mathrm{rEc} . \gamma \cdot \mathrm{dC} .46^{\prime} . \Delta \mathrm{tY}(\mathbf{\Delta})$ was inoculated into $1 \mathrm{~L}$ of permissive (+SAA/+L-arabinose, blue) or nonpermissive (-sAA/L-arabinose, red) LB media and incubated with agitation at $34^{\circ} \mathrm{C}$ for 20 days. Results from the equivalent experiment with the non-contained ancestor $\mathrm{rEc} . \gamma(\bullet)$ are also shown. Cultures were frequently monitored by a, $\mathrm{OD}_{600}$ and quantification of CFUs on solid b, permissive (+sAA/ $+_{\mathrm{L}}-$ arabinose) and c, nonpermissive (-sAA/-L-arabinose) LB media. CFUs are plotted as the average of three replicates. Open symbols indicate that no CFUs were observed. Symbols for $\mathrm{rEc} . \gamma \cdot \mathrm{dC} .46^{\prime} . \Delta \mathrm{tY}$ are not visible because CFUs were never observed from either permissive or nonpermissive liquid cultures plated on nonpermissive solid media. At the end of the 20-day growth period, both cultures containing $\mathrm{rEc} . \gamma \cdot \mathrm{dC} .46^{\prime} . \Delta \mathrm{tY}$ were interrogated for the presence of a single EM by plating each $1 \mathrm{~L}$ culture across 30 nonpermissive solid media plates. CFUs were not observed and remained below the limit of detection for the following seven-day observation period. We hypothesize that the decrease in CFU counts obtained on permissive solid media for the permissive culture of $\mathrm{rEc} . \gamma \cdot \mathrm{dC} .46^{\prime} . \Delta \mathrm{tY}$ reflects $\mathrm{pAzF}$ degradation at $\geq 6$ days. Reported CFUs are averages, where $n=3$ technical replicates, and error bars are \pm s.d. Refer to the methods for a complete description of this experiment. 


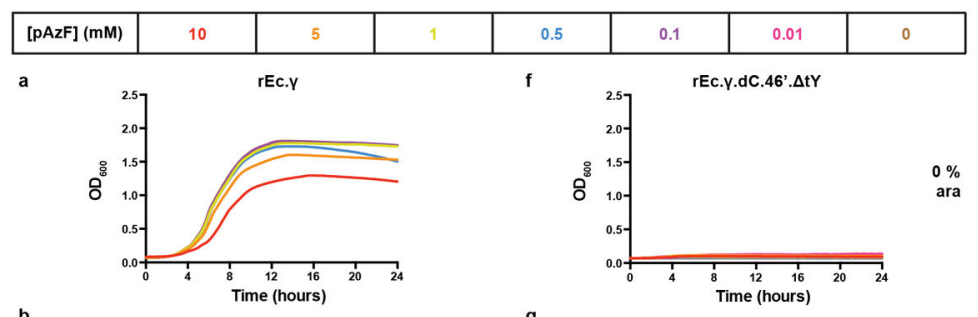

b
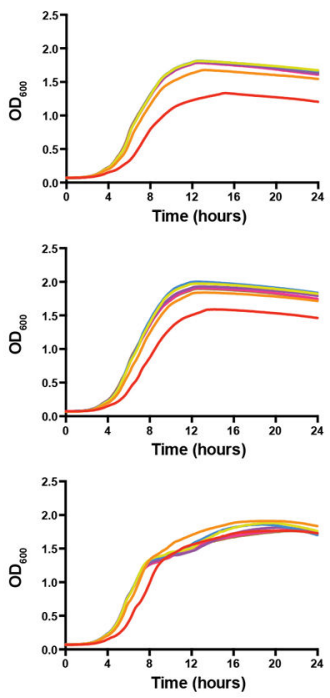

e
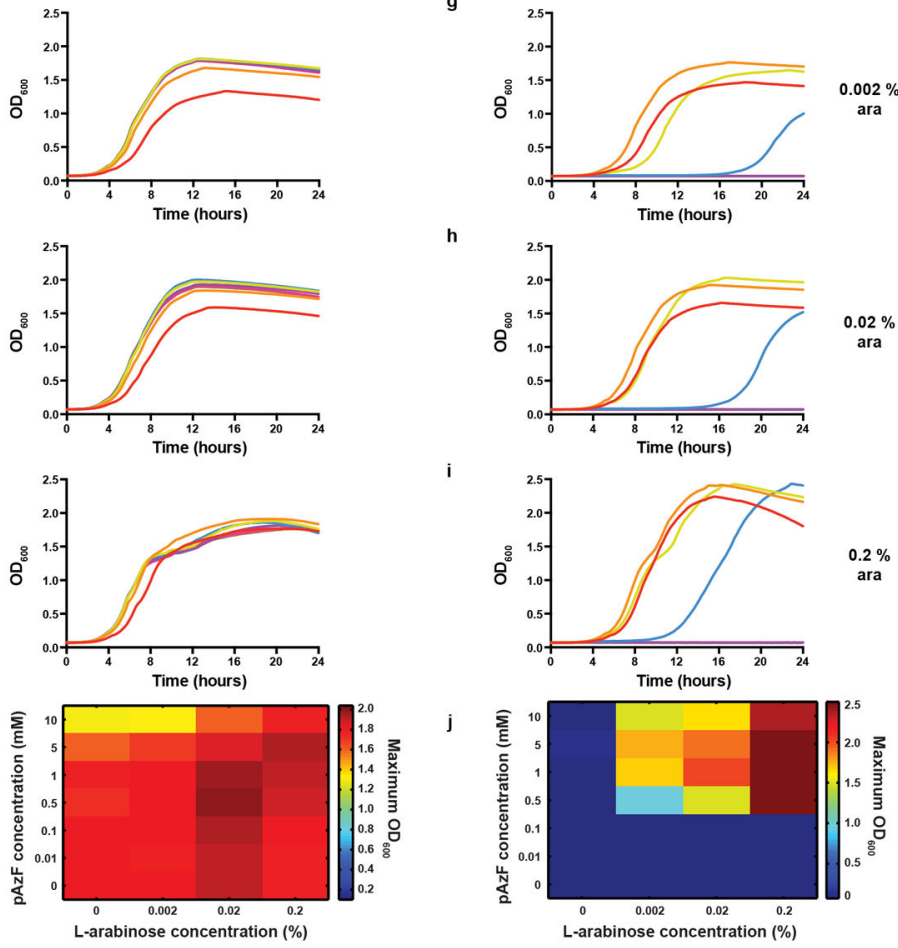

Extended Data Figure 7. Dose-dependent growth of rEc. $\gamma \cdot d C .46^{\prime} . \Delta t Y$ in pAzF and $\mathrm{L}$-arabinose compared to the non-contained ancestor

Growth in LB media supplemented with different concentrations of $\mathrm{pAzF}$ and $\mathrm{L}$-arabinose. Growth profiles for $\mathrm{rEc} . \gamma$ across a gradient of pAzF concentrations in the presence of $\mathbf{a}, 0 \%$, b, $0.002 \%, \mathbf{c}, 0.02 \%$, and d, $0.2 \%$ L-arabinose. Growth profiles for rEc. $\gamma \cdot \mathrm{dC} .46^{\prime} . \Delta \mathrm{tY}$ across a gradient of $\mathrm{pAzF}$ concentrations in the presence of $\mathbf{f}, 0 \%, \mathbf{g}, 0.002 \%, \mathbf{h}, 0.02 \%$, and $\mathbf{i}, 0.2 \%$ L-arabinose. $\mathbf{e}$ and $\mathbf{j}$, Growth profiles illustrated in parts a-d and f-i are depicted as heat maps in parts e and $\mathrm{j}$, respectively, where the maximum $\mathrm{OD}_{600}$ was obtained from the average of three replicates and plotted in MATLAB. Reported growth profiles and heat map values are averages, where $n=3$ technical replicates, and error bars are \pm s.d.

Nature. Author manuscript; available in PMC 2015 October 01. 


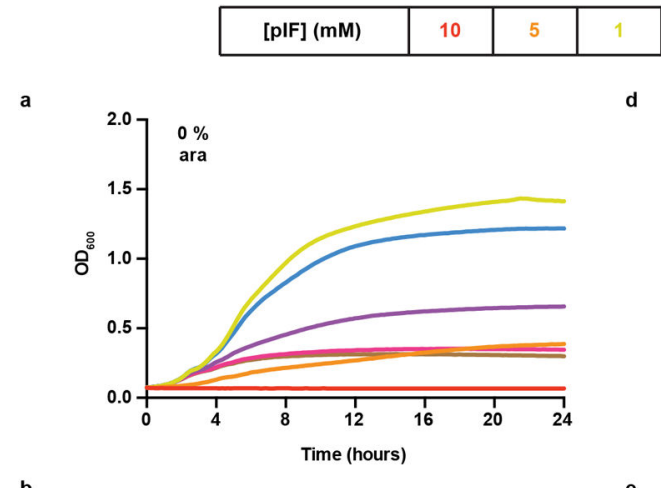

\begin{tabular}{l|l|l|l|}
0.5 & 0.1 & 0.01 & 0 \\
\hline
\end{tabular}

d

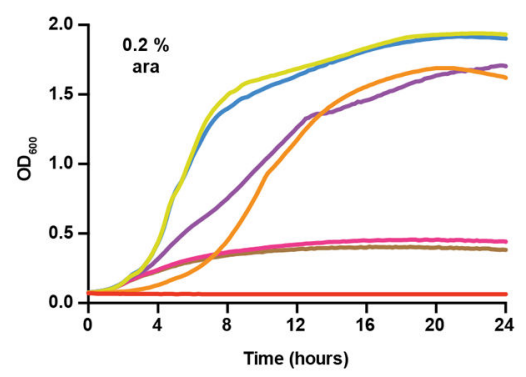

b

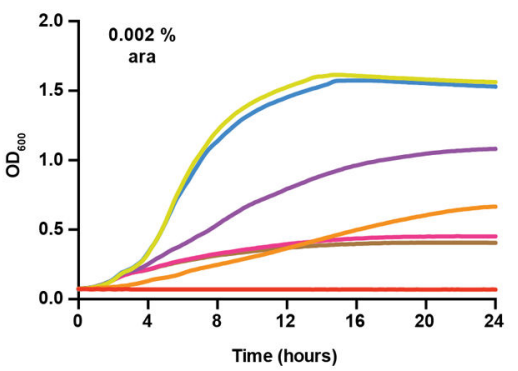

e

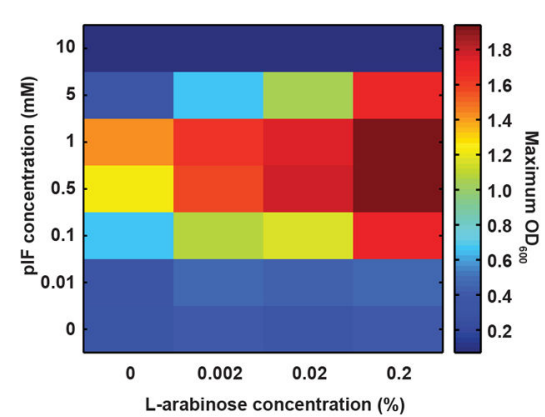

c

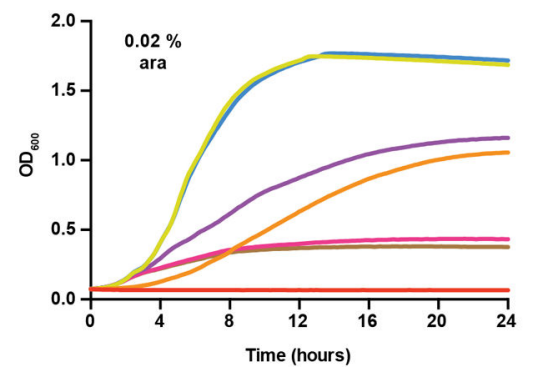

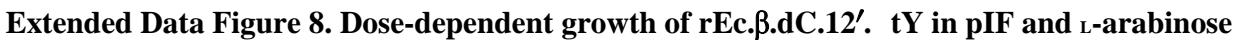
Growth in LB media supplemented with different concentrations of pIF and $\mathrm{L}_{\text {-arabinose. }}$ Growth profiles for $\mathrm{rEc} . \beta . \mathrm{dC} .12^{\prime} . \Delta \mathrm{tY}$ across a gradient of pIF concentrations in the presence of $\mathbf{a}, 0 \%, \mathbf{b}, 0.002 \%, \mathbf{c}, 0.02 \%$, and $\mathbf{d}, 0.2 \%$ L-arabinose. e, Growth profiles illustrated in parts a-d are depicted as a heat map, where the maximum $\mathrm{OD}_{600}$ was obtained from the average of three replicates and plotted in MATLAB. Reported growth profiles and heat map values are averages, where $n=3$ technical replicates, and error bars are \pm s.d. 


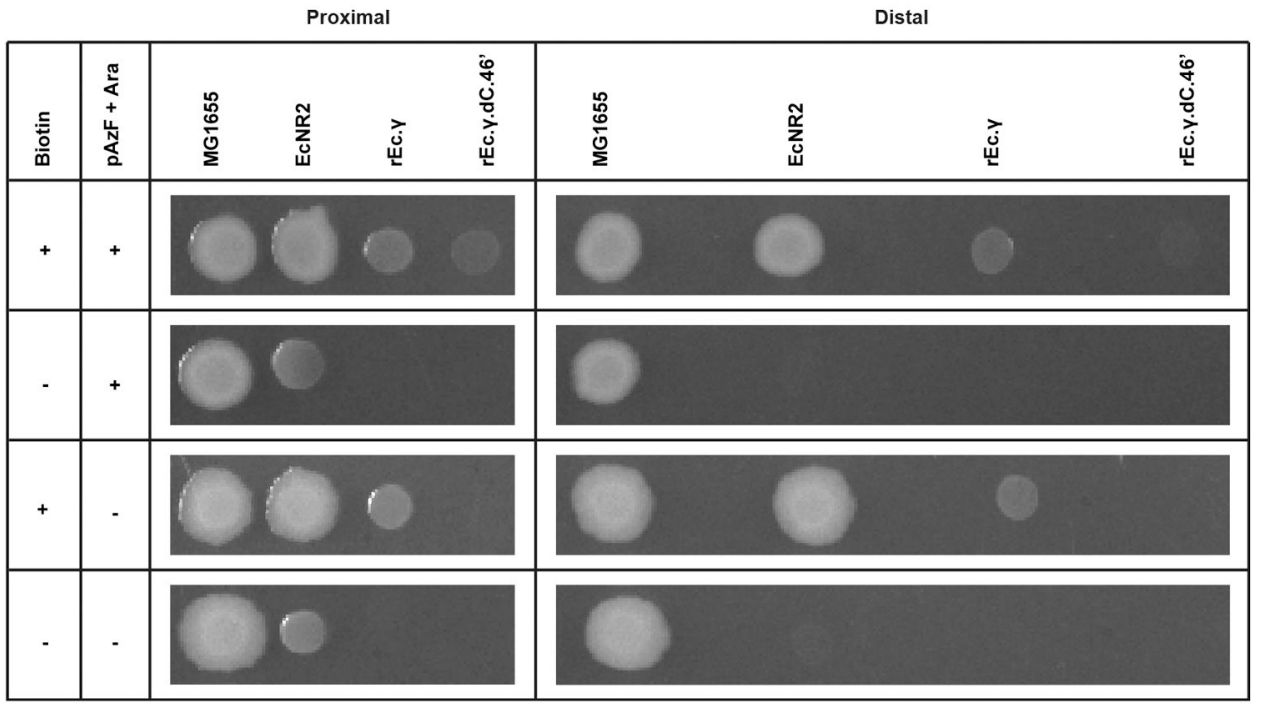

Extended Data Figure 9. Proximity-dependent complementation of biotin auxotrophy Wild type E. coli K-12 substr. MG1655 and three strains auxotrophic for biotin, EcNR2, rEc. $\gamma$ (a non-contained GRO with an integrated pAzF OTS), and rEc. $\gamma \cdot d C .46^{\prime}$ (also a synthetic auxotroph) were grown either adjacent or separately on rich-defined solid media. EcNR2 grew on biotin-deficient media when plated in close proximity to wild type E. coli, suggesting cross-feeding of the essential metabolite. The pAzF auxotroph only grew on media supplemented with biotin, $\mathrm{pAzF}$, and $\mathrm{L}$-arabinose.

\section{Supplementary Material}

Refer to Web version on PubMed Central for supplementary material.

\section{Acknowledgments}

We are grateful to D. Söll and Y.S. Wang for inspired discussion and for providing pTech-supU. We thank N. Carriero and R. Bjornson at the Yale Biomedical High Performance Computing Cluster for assistance with SIFT. Funding was from Defense Advanced Research Projects Agency (N66001-12-C-4020, N66001-12-C-4211); NIHMSTP-TG-T32GM07205 (A.D.H.); DuPont Inc. and the Arnold and Mabel Beckman Foundation (F.J.I.).

\section{References}

1. Way JC, Collins JJ, Keasling JD, Silver PA. Integrating Biological Redesign: Where Synthetic Biology Came From and Where It Needs to Go. Cell. 2014; 157:151-161.10.1016/j.cell. 2014.02.039 [PubMed: 24679533]

2. Schmidt M, de Lorenzo V. Synthetic constructs in/for the environment: managing the interplay between natural and engineered Biology. FEBS letters. 2012; 586:2199-2206.10.1016/j.febslet. 2012.02.022 [PubMed: 22710182]

3. Moe-Behrens GH, Davis R, Haynes KA. Preparing synthetic biology for the world. Frontiers in microbiology. 2013; 4:5.10.3389/fmicb.2013.00005 [PubMed: 23355834]

4. Pieper DH, Reineke W. Engineering bacteria for bioremediation. Current opinion in biotechnology. 2000; 11:262-270. [PubMed: 10851148]

5. Steidler L. Genetically engineered probiotics. Best practice \& research Clinical gastroenterology. 2003; 17:861-876. [PubMed: 14507594]

6. Kong W, et al. Regulated programmed lysis of recombinant Salmonella in host tissues to release protective antigens and confer biological containment. Proceedings of the National Academy of 
Sciences of the United States of America. 2008; 105:9361-9366.10.1073/pnas.0803801105 [PubMed: 18607005]

7. Szafranski P, et al. A new approach for containment of microorganisms: dual control of streptavidin expression by antisense RNA and the T7 transcription system. Proceedings of the National Academy of Sciences of the United States of America. 1997; 94:1059-1063. [PubMed: 9037005]

8. Steidler L, et al. Biological containment of genetically modified Lactococcus lactis for intestinal delivery of human interleukin 10 . Nature biotechnology. 2003; 21:785-789.10.1038/nbt840

9. Ronchel MC, Ramos JL. Dual system to reinforce biological containment of recombinant bacteria designed for rhizoremediation. Applied and environmental microbiology. 2001; 67:26492656.10.1128/AEM.67.6.2649-2656.2001 [PubMed: 11375176]

10. Jensen LB, Ramos JL, Kaneva Z, Molin S. A substrate-dependent biological containment system for Pseudomonas putida based on the Escherichia coli gef gene. Applied and environmental microbiology. 1993; 59:3713-3717. [PubMed: 8285679]

11. Lajoie MJ, et al. Genomically recoded organisms expand biological functions. Science. 2013; 342:357-360.10.1126/science.1241459 [PubMed: 24136966]

12. Wang HH, et al. Programming cells by multiplex genome engineering and accelerated evolution. Nature. 2009; 460:894-898.10.1038/nature08187 [PubMed: 19633652]

13. Marchler-Bauer A, et al. CDD: a Conserved Domain Database for protein classification. Nucleic acids research. 2005; 33:D192-196.10.1093/nar/gki069 [PubMed: 15608175]

14. Cohen SN, Chang AC, Boyer HW, Helling RB. Construction of biologically functional bacterial plasmids in vitro. Proceedings of the National Academy of Sciences of the United States of America. 1973; 70:3240-3244. [PubMed: 4594039]

15. Berg P, Baltimore D, Brenner S, Roblin RO, Singer MF. Summary statement of the Asilomar conference on recombinant DNA molecules. Proceedings of the National Academy of Sciences of the United States of America. 1975; 72:1981-1984. [PubMed: 806076]

16. NIH Guidelines for research involving recombinant or synthetic DNA molecules. Office of Biotechnology Activities; Bethesda, MD: 2013.

17. Betenbaugh MJ, Beaty C, Dhurjati P. Effects of plasmid amplification and recombinant gene expression on the growth kinetics of recombinant E. coli. Biotechnology and bioengineering. 1989; 33:1425-1436.10.1002/bit.260331110 [PubMed: 18587883]

18. Sorensen SJ, Bailey M, Hansen LH, Kroer N, Wuertz S. Studying plasmid horizontal transfer in situ: a critical review. Nature reviews Microbiology. 2005; 3:700-710.10.1038/nrmicro1232 [PubMed: 16138098]

19. Kumar P, Henikoff S, Ng PC. Predicting the effects of coding non-synonymous variants on protein function using the SIFT algorithm. Nature protocols. 2009; 4:1073-1081.10.1038/nprot.2009.86 [PubMed: 19561590]

20. Schaaper RM, Dunn RL. Spectra of spontaneous mutations in Escherichia coli strains defective in mismatch correction: the nature of in vivo DNA replication errors. Proceedings of the National Academy of Sciences of the United States of America. 1987; 84:6220-6224. [PubMed: 3306672]

21. Eggertsson G, Soll D. Transfer ribonucleic acid-mediated suppression of termination codons in Escherichia coli. Microbiological reviews. 1988; 52:354-374. [PubMed: 3054467]

22. Maisnier-Patin S, Berg OG, Liljas L, Andersson DI. Compensatory adaptation to the deleterious effect of antibiotic resistance in Salmonella typhimurium. Molecular microbiology. 2002; 46:355366. [PubMed: 12406214]

23. Holberger LE, Hayes CS. Ribosomal protein S12 and aminoglycoside antibiotics modulate A-site mRNA cleavage and transfer-messenger RNA activity in Escherichia coli. The Journal of biological chemistry. 2009; 284:32188-32200.10.1074/jbc.M109.062745 [PubMed: 19776006]

24. Abe Y, et al. Structure and function of DnaA N-terminal domains: specific sites and mechanisms in inter-DnaA interaction and in DnaB helicase loading on oriC. The Journal of biological chemistry. 2007; 282:17816-17827.10.1074/jbc.M701841200 [PubMed: 17420252]

25. Bosl M, Kersten H. A novel RNA product of the tyrT operon of Escherichia coli. Nucleic acids research. 1991; 19:5863-5870. [PubMed: 1840671]

26. O'Donoghue P, Ling J, Wang YS, Soll D. Upgrading protein synthesis for synthetic biology. Nature chemical biology. 2013; 9:594-598.10.1038/nchembio.1339 [PubMed: 24045798] 
27. Lajoie MJ, et al. Probing the limits of genetic recoding in essential genes. Science. 2013; 342:361363.10.1126/science.1241460 [PubMed: 24136967]

28. Bain JD, Switzer C, Chamberlin AR, Benner SA. Ribosome-mediated incorporation of a nonstandard amino acid into a peptide through expansion of the genetic code. Nature. 1992; 356:537539.10.1038/356537a0 [PubMed: 1560827]

29. Pinheiro VB, et al. Synthetic genetic polymers capable of heredity and evolution. Science. 2012; 336:341-344.10.1126/science.1217622 [PubMed: 22517858]

30. Hammerling MJ, et al. Bacteriophages use an expanded genetic code on evolutionary paths to higher fitness. Nature chemical biology. 2014; 10:178-180.10.1038/nchembio.1450 [PubMed: 24487692]

31. Normanly J, Masson JM, Kleina LG, Abelson J, Miller JH. Construction of two Escherichia coli amber suppressor genes: tRNAPheCUA and tRNACysCUA. Proceedings of the National Academy of Sciences of the United States of America. 1986; 83:6548-6552. [PubMed: 3529087]

32. Gibson DG, et al. Enzymatic assembly of DNA molecules up to several hundred kilobases. Nature methods. 2009; 6:343-345.10.1038/nmeth.1318 [PubMed: 19363495]

33. Fan C, Ho JM, Chirathivat N, Soll D, Wang YS. Exploring the substrate range of wild-type aminoacyl-tRNA synthetases. Chembiochem: a European journal of chemical biology. 2014; 15:1805-1809.10.1002/cbic.201402083 [PubMed: 24890918]

34. Carr PA, et al. Enhanced multiplex genome engineering through co-operative oligonucleotide coselection. Nucleic acids research. 2012; 40:e132.10.1093/nar/gks455 [PubMed: 22638574]

35. Young TS, Ahmad I, Yin JA, Schultz PG. An enhanced system for unnatural amino acid mutagenesis in E. coli. Journal of molecular biology. 2010; 395:361-374.10.1016/j.jmb. 2009.10.030 [PubMed: 19852970]

36. Baba T, et al. Construction of Escherichia coli K-12 in-frame, single-gene knockout mutants: the Keio collection. Molecular systems biology. 2006; 2:20060008.10.1038/msb4100050

37. Richmond CS, Glasner JD, Mau R, Jin H, Blattner FR. Genome-wide expression profiling in Escherichia coli K-12. Nucleic acids research. 1999; 27:3821-3835. [PubMed: 10481021]

38. Isaacs FJ, et al. Precise manipulation of chromosomes in vivo enables genome-wide codon replacement. Science. 2011; 333:348-353.10.1126/science.1205822 [PubMed: 21764749]

39. Sharan SK, Thomason LC, Kuznetsov SG, Court DL. Recombineering: a homologous recombination-based method of genetic engineering. Nature protocols. 2009; 4:206-223.10.1038/ nprot.2008.227 [PubMed: 19180090]

40. Krzywinski M, et al. Circos: an information aesthetic for comparative genomics. Genome research. 2009; 19:1639-1645.10.1101/gr.092759.109 [PubMed: 19541911]

41. Rinehart J, et al. WNK2 kinase is a novel regulator of essential neuronal cation-chloride cotransporters. The Journal of biological chemistry. 2011; 286:30171-30180.10.1074/ jbc.M111.222893 [PubMed: 21733846]

42. Rappsilber J, Ishihama Y, Mann M. Stop and go extraction tips for matrix-assisted laser desorption/ionization, nanoelectrospray, and LC/MS sample pretreatment in proteomics. Analytical chemistry. 2003; 75:663-670. [PubMed: 12585499]

43. Heinemann IU, et al. Enhanced phosphoserine insertion during Escherichia coli protein synthesis via partial UAG codon reassignment and release factor 1 deletion. FEBS letters. 2012; 586:37163722.10.1016/j.febslet.2012.08.031 [PubMed: 22982858]

44. Neidhardt FC, Bloch PL, Smith DF. Culture medium for enterobacteria. Journal of bacteriology. 1974; 119:736-747. [PubMed: 4604283] 
a. Essential gene targeting strategies

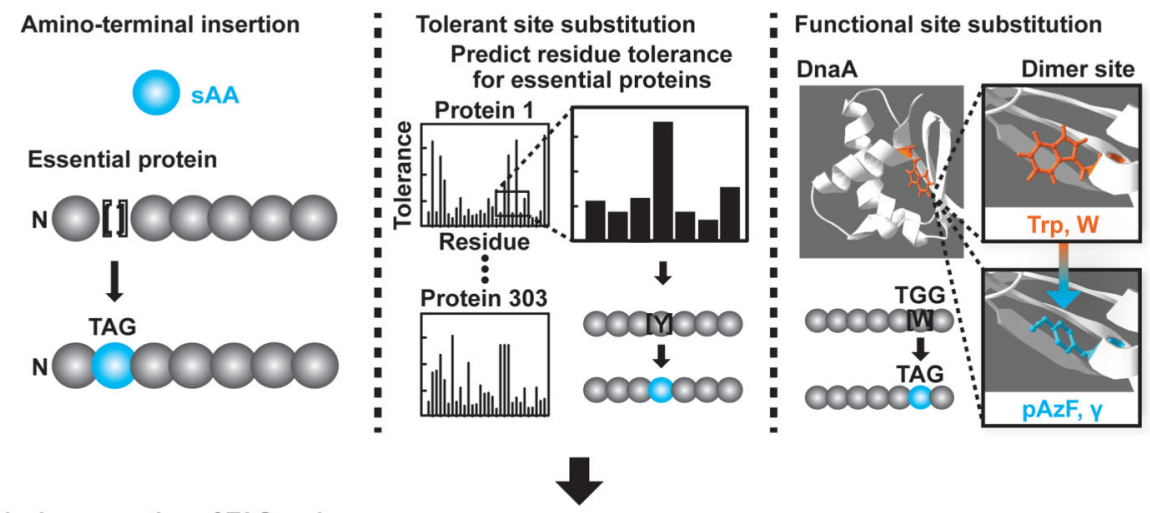

b. Incorporation of TAG codons Mutagenic ssDNA oligonucleotides
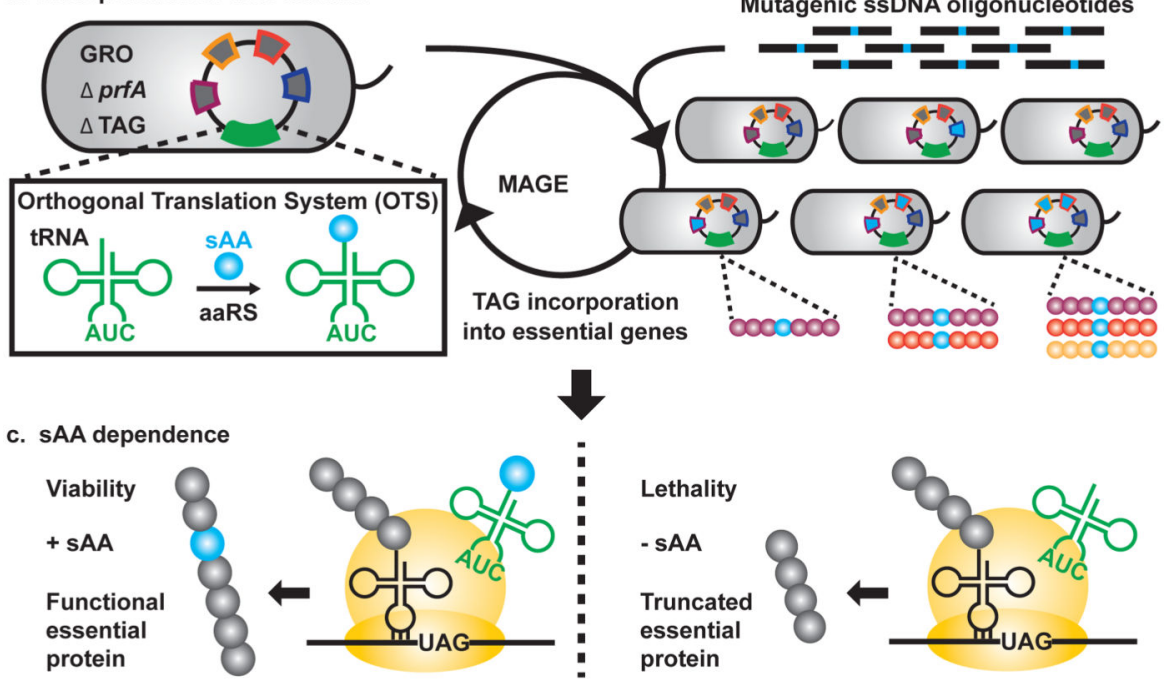

Figure 1. Strategy used to engineer GROs to require sAAs for growth

a, Approaches used to identify suitable loci within essential proteins for synthetic amino acid (sAA, blue) incorporation. b, MAGE was used for site-specific incorporation of TAG codons into essential genes of a genomically recoded organism (GRO) lacking all natural TAG codons ( $\triangle \mathrm{TAG})$ and release factor 1 ( $\Delta p r f A)$, and containing an orthogonal translation system (OTS, green) consisting of the $M$. jannaschii aminoacyl-tRNA synthetase (aaRS) and cognate UAG-decoding tRNA. c, Synthetic auxotrophs that depend on sAAs for growth were isolated. 

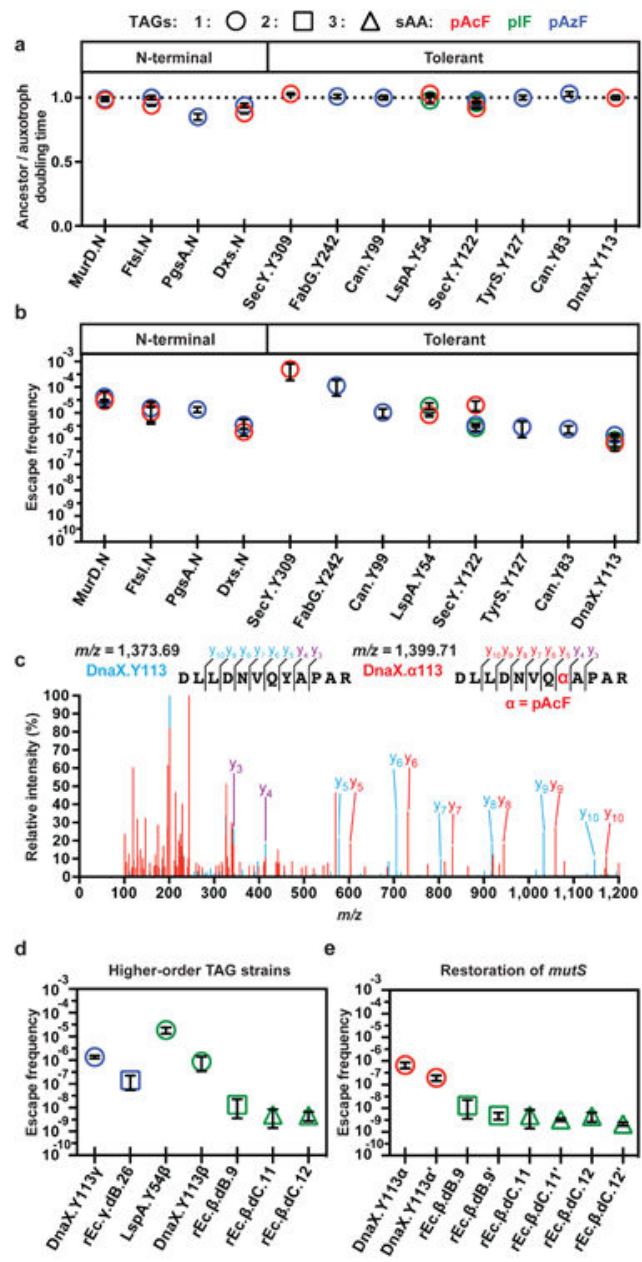

Figure 2. Characterization of strains dependent on SAA incorporation in essential proteins a, Doubling time (DT) ratios for the non-contained ancestor to synthetic auxotroph containing one TAG. b, Escape frequencies (EFs) of strains from a. c, Superimposed MS/MS spectra for DnaX peptides from DnaX.Y113a (red) and the non-contained ancestor rEc.a (blue). Overlapping peaks are purple and a mass shift relative to rEc.a identifies Y113 as the pAcF incorporation site in DnaX.Y113a; see methods. d, EFs for strains with multiple TAG codons e, and/or functional mismatch repair (prime, $m u t S^{+}$). For all plots, average values of 3 technical replicates are plotted with error bars, \pm s.d. 

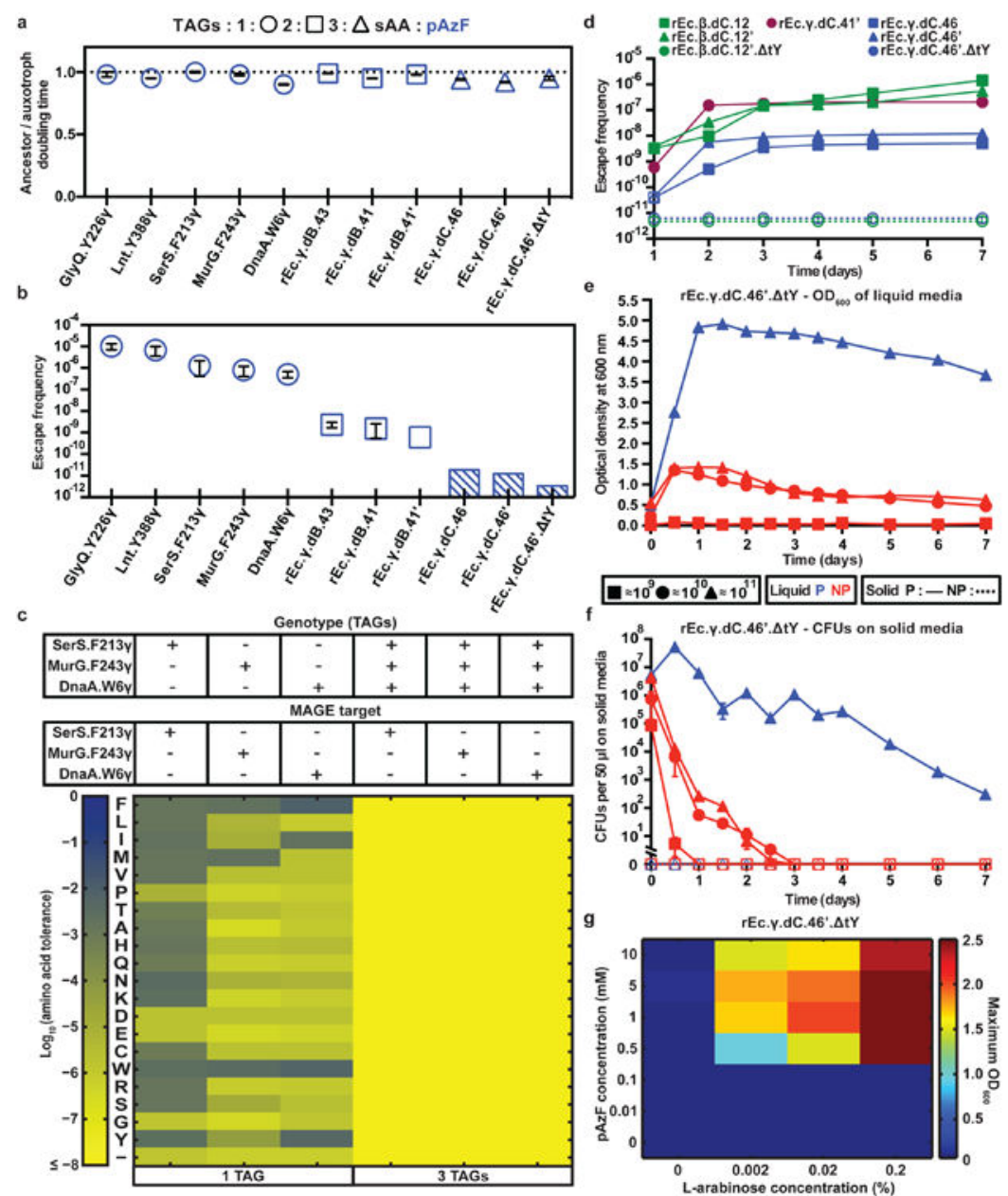

Figure 3. Characterization of strains dependent on SAA incorporation at active and dimerization sites in essential proteins

a, Doubling time (DT) ratios for the non-contained ancestor to pAzF auxotroph with one or more TAGs at functional loci calculated from growth in $5 \mathrm{mM} \mathrm{pAzF}$ and $0.2 \% \mathrm{~L}$-arabinose.

b, Escape frequencies (EFs) of strains in part a; bars represent EFs below the detection limit; average EFs are plotted. c, Representative assay surveying tolerance of TAG loci to 20 amino acids in different synthetic auxotrophs and expressed as $\log _{10}$ of total cell survival; + indicates a TAG codon at the locus in the background strain and - indicates the wild type codon; blue and yellow indicate high and low tolerance to substitution, respectively; see methods. d, Representative escape assay monitoring EFs up to seven days after plating on solid nonpermissive media; hollow symbols/dashed lines, no observed EMs; see methods. e, Temporal monitoring of permissive ( $\mathrm{P}$, blue) and nonpermissive (NP, red) cultures inoculated with $\sim 10^{9}, 10^{10}$, or $10^{11}$ cells of rEc. $\gamma \cdot \mathrm{dC} .46^{\prime} . \Delta \mathrm{tY}$ by $\mathrm{OD}_{600}$ and $\mathbf{f}$, associated CFUs as sampled on P (solid lines) or NP (dashed lines) solid media; CFUs were never observed on NP solid media; hollow data points indicate no observed CFUs. g, Maximum $\mathrm{OD}_{600}$ values during growth in LB across a concentration gradient of pAzF and $\mathrm{L}$-arabinose. For all plots, average values of 3 technical replicates are plotted with error bars, \pm s.d. 


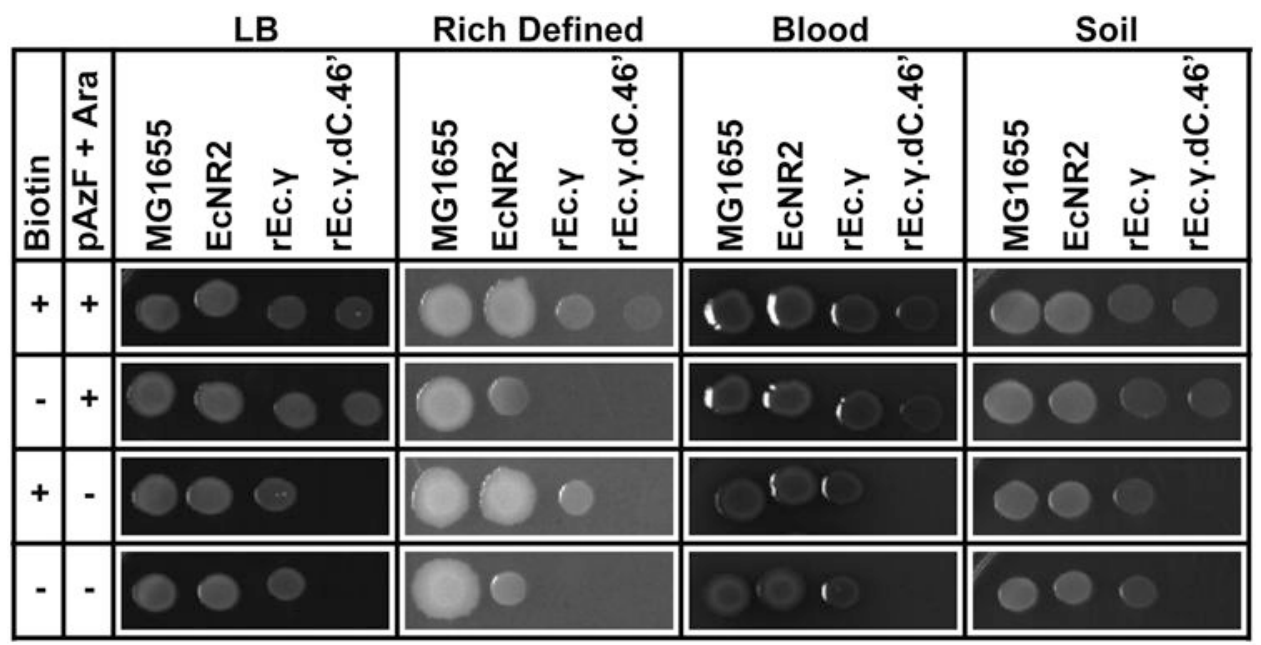

Figure 4. Investigating the viability of synthetic auxotrophs on diverse media types Rescue by cross-feeding shown through spotting on diverse media types $+/-\mathrm{pAzF} / \mathrm{L}-$ arabinose and biotin supplementation; EcNR2, $\mathrm{rEc} . \gamma$, and $\mathrm{rEc} . \gamma \cdot \mathrm{dC} .46^{\prime}$ are auxotrophic for biotin $(\triangle b i o A / B)$ and rEc. $\gamma . \mathrm{dC} .46^{\prime}$ is also a pAzF auxotroph. 